\title{
Systematic analysis of the role of SLC52A2 in multiple human cancers
}

\author{
Lilong Zhang ${ }^{1,2 \dagger}$, Man $\mathrm{Li}^{1,2+}$, Zhoujun Cui ${ }^{3}$, Dongqi Chai ${ }^{1,2}$, Yongjun Guan ${ }^{1,2}$, Chen Chen ${ }^{1 *}$ and \\ Weixing Wang ${ }^{1 *}$ (D)
}

\begin{abstract}
Background: In humans, riboflavin must be obtained through intestinal absorption because it cannot be synthesized by the body. SLC52A2 encodes a membrane protein belonging to the riboflavin transporter protein family and is associated with a variety of diseases. Here, we systematically explore its relevance to multiple human tumors.

Methods: We analyzed the association of SLC52A2 with 33 tumors using publicly available databases such as TCGA and GEO. We verified the SLC52A2 expression in hepatocellular carcinoma, gastric cancer, colon cancer, and rectal cancer using immunohistochemistry.

Results: We report that SLC52A2 was highly expressed in almost all tumors, and the immunohistochemical results in the hepatocellular, gastric, colon, and rectal cancers were consistent with the above. SLC52A2 expression was linked to patient overall survival, disease-specific survival, progression-free interval, diagnosis, mutations, tumor mutational burden, microsatellite instability, common immune checkpoint genes, and immune cells infiltration. Enrichment analysis showed that SLC52A2 was mainly enriched in oocyte meiosis, eukaryotic ribosome biogenesis, and cell cycle. In hepatocellular carcinoma, the SLC52A2 expression is an independent prognostic factor. The SNHG3 and THUMPD3AS1/hsa-miR-139-5p-SLC52A2 axis were identified as potential regulatory pathways in hepatocellular carcinoma.
\end{abstract}

Conclusion: In conclusion, we have systematically described for the first time that SLC52A2 is closely associated with a variety of tumors, especially hepatocellular carcinoma.

Keywords: SLC52A2, Pan-cancer, Hepatocellular, Carcinoma, Bioinformatics, Prognosis

\section{Introduction}

Riboflavin (also called vitamin B2) is a water-soluble vitamin found in various foods. Flavin adenine dinucleotide and flavin mononucleotide are their most important biologically active forms, which are key to carbohydrates, amino acids, and lipid metabolism [1]. And riboflavin deficiency causes growth impairment [2]. Bacteria, fungi, and plants can synthesize riboflavin from GTP and

\footnotetext{
*Correspondence: appreciation@whu.edu.cn;Wangwx@whu.edu.cn ${ }^{\dagger}$ Lilong Zhang and Man Li contributed equally to this work

1 Department of General Surgery, Renmin Hospital of Wuhan University, No. 238, Jiefang Road, Wuchang District, Wuhan 430060, Hubei Province, China

Full list of author information is available at the end of the article
}

ribulose 5Pi. On the contrary, higher organisms lose the ability to synthesize this vitamin and must be obtained by intestinal absorption [3]. Riboflavin is absorbed mainly in the small intestine. Milk, dairy products, meat, fish, and dark-green vegetables are essential sources of riboflavin [4].

It was found that the absorption of riboflavin and regulation of riboflavin tissue distribution was mainly dependent on specific transporters of the human solute carrier family 52 (SLC52) [5]. Currently, this family has been confirmed to include SLC52A1, SLC52A2, SLC52A3 [5]. SLC52A2 (also known as GPR172A, RFT3, RFVT2, PAR1) gene, located at locus $8 \mathrm{q} 24.3$, encodes a protein of 445-amino acid [6-8]. SLC52A2-mediated riboflavin uptake has been confirmed to be $\mathrm{Na}^{+}$-independent,

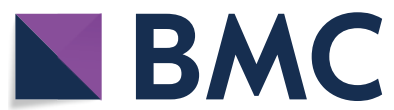

(c) The Author(s) 2022. Open Access This article is licensed under a Creative Commons Attribution 4.0 International License, which permits use, sharing, adaptation, distribution and reproduction in any medium or format, as long as you give appropriate credit to the original author(s) and the source, provide a link to the Creative Commons licence, and indicate if changes were made. The images or other third party material in this article are included in the article's Creative Commons licence, unless indicated otherwise in a credit line to the material. If material is not included in the article's Creative Commons licence and your intended use is not permitted by statutory regulation or exceeds the permitted use, you will need to obtain permission directly from the copyright holder. To view a copy of this licence, visit http://creativecommons.org/licenses/by/4.0/. The Creative Commons Public Domain Dedication waiver (http://creativeco mmons.org/publicdomain/zero/1.0/) applies to the data made available in this article, unless otherwise stated in a credit line to the data. 
$\mathrm{Cl}^{-}$-independent, and $\mathrm{pH}$-independent, which has been indicated that SLC52A2 is crucial for tissue distribution of riboflavin $[7,9]$.

It has been found that the organism is susceptible to riboflavin deficiency under pathological stress. Such riboflavin deficiency has been considered a risk factor for cancer, cardiovascular disease, and neurodegeneration [1]. Cheng et al. firstly reported the study of cancerrelated copy number amplification and overexpression of SLC52A2. They found that, compared with matched adjacent noncancerous, SLC52A2 showed increased copy number in $44 \%$ gastric cancer tissues and its expression was upregulated in 56\% gastric cancer tissues [10].

In this study, bioinformatic analysis was conducted to explore the SLC52A2 expression in various human tumors and its possible association with cancer, which was confirmed by immunohistochemistry, to provide more information to better understand the importance of SLC52A2 in various cancers.

\section{Materials and methods Data collection and processing}

Xena Browser was used to obtain the Genotype-Tissue Expression (GTEx) data and The Cancer Genome Atlas (TCGA) data. The Cancer Cell Line Encyclopedia (CCLE) database was used to acquire the SLC52A2 expression in cancer cell lines. The ONCOMINE database was selected to further understand the SLC52A2 expression in multiple cancers, with "p-value $=0.05$ " and "fold change $=1.5$ ". The TISIDB database was used to examine SLC52A2 expression in different immune subtypes in tumor samples [11]. The cBioPortal database and Catalogue of Somatic Mutations in Cancer (COSMIC) database were used to estimate the mutation levels of SLC52A2 in different human cancers [12, 13]. GSE121248, GSE112790, GSE39791, GSE55092, GSE62232, and GSE102079 datasets were downloaded via GEO. Tumor IMmune Estimation Resource (TIMER) was used to estimate the association between SLC52A2 and immune cell infiltration [14].

\section{mRNA expression of SLC52A2 in multiple human cancers}

The "gganatogram" and "ggpubr" R software packages were used to study the SLC52A2 expression in 31 normal human tissues. And, unpaired t-test was conducted to estimate the differences between males and females in normal tissues. For all TCGA data, the number of normal samples per tumor was counted, and tumors with the number of normal samples $\geq 5$ were selected. Then, the 'ggpubr' $\mathrm{R}$ software package was used to estimate the expression of differences by Wilcoxon's test between normal and tumor tissues. Besides, we also investigated the association between clinical stages, grade, gender, race, tumor status, and SLC52A2 expression in 33 cancers by "limma" and "ggpubr" R software packages.

\section{Cox regression analysis, survival analysis, and diagnosis analysis}

The association between SLC52A2 expression and overall survival (OS), disease-specific survival (DSS), progression-free interval (PFI) was assessed using phenotype and survival data of TCGA data. The difference in survival between the high and low expression groups was estimated by the Kaplan-Meyer method using the "survival" and "survivor" $\mathrm{R}$ packages. The Cox proportional hazard regression analysis was used to evaluate the hazard ratio by "survival", "survminer", "limmu" and "forestplot" R software package. Also, the AUC value was calculated by using the "pROC" R software package.

\section{Tumor mutational burden and microsatellite instability}

To calculate the number of mutations in the 33 cancer samples, the tumor mutational burden (TMB) was estimated and divided by the total length of the exons. The microsatellite instability (MSI) score was acquired from TCGA. We used Spearman's method to estimate the correlation analysis between the SLC52A2 expression and TMB or MSI. Both indicators were displayed on a radar map by the "fmsb" R software package.

\section{Immune cell infiltration enrichment and co-expression analysis of some common immune checkpoint genes} Co-expression analysis of immune marker set and some common immune checkpoint genes was conducted with "limma" $\mathrm{R}$ software packages and visualized using "reshape2" and "RColorBrewer" R software packages. On the TIMER2.0 platform [15], we used CIBERSORT and TIDE algorithms to analyze the relationship between SLC52A2 expression and immune cell infiltration.

\section{SLC52A2-related gene enrichment analysis}

The "Similar Gene Detection" module of GEPIA2 was used to acquire the SLC52A2-related genes $(R>0.4)$. Then, the protein interaction network of the above genes was established using the STRING website [16]. Both Kyoto Encyclopedia of Genes and Genomes (KEGG) and Gene Ontology (GO) enrichment pathway as well as functional annotation analysis of SLC52A2 in 33 types of tumors were conducted using "clusterProfiler”, “org.Hs.eg.db”, “enrichplot”, “DOSE”, “colorspace”, "stringi" and "ggplot2" R software package. In addition, according to the expression level of SLC52A2, patients in the LIHC-TCGA cohort were divided into high and low expression groups, and then KEGG (c2.cp.kegg. v7.4.symbols.gmt) and GO (c5.go.bp.v7.4.symbols.gmt, c5.go.cc.v7.4.symbols.gm-t, c5.go.mf.v7.4.symbols.gmt) 
enrichment analysis was performed using GSEA_4.1.0 software.

\section{Prognostic analysis of SLC52A2 in LIHC}

We conducted univariate and multivariate Cox regression analyses to determine the appropriate terms for constructing the nomogram by TCGA database. Forests were used to display the P-value, HR, and 95\% confidence interval for each variable by "forestplot" $\mathrm{R}$ software package. According to the results of a multivariate Cox proportional hazards analysis, a nomogram was developed to predict the 1-, 2-, 3- and 5-year OS.

\section{Prediction of upstream IncRNA and miRNAs of SLC52A2 in LIHC}

starBase is a database for exploring miRNA-related studies [17]. Upstream binding miRNAs of SLC52A2 were predicted using the miRNA-Target module (miRNAmRNA option), and the lncRNAs upstream of hsa-miR139-5p were explored using the miRNA-Target module (miRNA-lncRNA option) in starbase. Pan-cancer module in starbase was used to analyze the miRNA differential expression, miRNA survival analysis, and miRNA-target coexpression. The GEPIA2 tool was used to estimate the lncRNA differential expression and lncRNA survival analysis.

\section{Immunohistochemical staining}

We collected tumor and paraneoplastic tissues from LIHC (40 cases), STAD (20 cases), COAD (20 cases), and READ (20 cases) from January 2018 to December 2020 at the Renmin Hospital of Wuhan University, China. Immunohistochemistry analysis was performed on these cancers. Briefly, the paraffin sections were placed on a stainless shelf and incubated at $70{ }^{\circ} \mathrm{C}$ for $80 \mathrm{~min}$ in an oven. After xylene deparaffinization twice (20 min each), gradient ethanol hydration $(100 \%, 95 \%, 85 \%, 75 \%, 5 \%$, 5 min each), and deionized water washing. The sections were put on boiling of sodium citrate buffer $(\mathrm{pH} 9.0)$ and further boiled for $4 \mathrm{~min}$ in a pressure cooker for antigen retrieval. After cooling, the slides were blocked with 3\% hydrogen peroxide solution for $15 \mathrm{~min}$. The sections were incubated overnight at $4{ }^{\circ} \mathrm{C}$ with anti-SLC52A2 antibody (rabbit, Solarbio, 1:100) in a humidity box, then incubated with a second antibody. The staining results were visualized using the 3,5-diaminobenzidine. Immunostained sections were characterized quantitatively as the integrated optical density (IOD) by digital image analysis using the Image 1.53e software (Wayne Rasband and contributors National Institutes of Health, USA). Images were obtained with an fluorescence microscope (Olympus BX63, Tokyo, Japan). A series of 5 random images on each section was taken for each immunostained parameter to obtain a mean value for statistical comparison. The cutoff for the definition of high expression group or low expression group was the median value. Data were expressed as the mean \pm standard deviation. Statistical measurements were performed using the SPSS 21.0 statistical software (SPSS Inc., Chicago, USA).

\section{Quantitative reverse transcription PCR (qRT-PCR)}

Among the above LIHC patients, 15 of them had their tumor tissues and paraneoplastic tissues frozen at $-80{ }^{\circ} \mathrm{C}$. Therefore, we used this part of patients for the detection of the SLC52A2 mRNA level. Total RNA was extracted using Trizol RNA extraction kit (Servicebio) according to the manufacture's instructions. RNA was reverse-transcribed to complementary DNA according to the manufacturer's instructions of Revert Aid First Strand cDNA Synthesis Kit (Thermo Fisher Scientific). qRT-PCR was performed with the primers for SLC52A2 (F: AGG TGGGAAAAGAACTGGC; R: CAAGCACAGAGACGT AAGAGG) (Tsingke Biotechnology Co., Ltd.) and $\beta$-actin (F: CCTTCCTGGGCATGGAGTC; R: TGATCTTCA TTGTGCTGGGTG) (Tsingke Biotechnology Co., Ltd.) using $2 \times$ SYBR Green qPCR Master Mix (None ROX) (Servicebio). The reaction conditions were pre-denaturation at $95^{\circ} \mathrm{C}$ for $30 \mathrm{~s}$, followed by 40 cycles of denaturation at $95{ }^{\circ} \mathrm{C}$ for $15 \mathrm{~s}$ and annealing/extension at $60{ }^{\circ} \mathrm{C}$ for $30 \mathrm{~s}$. The relative gene expression level was computed by the $2^{-\Delta \Delta \mathrm{CT}}$ way. $\beta$-actin was used as an internal reference for normalization.

See Additional file 1: Table S1 for details of the database sites used in this study. Table 1 provided the full cancer type name corresponding to each abbreviation listed in the legend and the text.

\section{Results}

mRNA expression of SLC52A2 in multiple human cancers

First, we used the GTEx database to analyze the SLC52A2 expression in 31 types of normal tissues. The results revealed that the SLC52A2 expression was the highest in the bone marrow, spleen and testis tissues, while the lowest in the skeletal muscle, blood, and liver (Fig. 1A). Besides, the expression of SLC52A2 in adipose tissues and skeletal muscle tissues was significantly higher in females than in males (Fig. 1B, C; Additional file 4: Fig. S1A). Subsequently, the CCLE database was used to estimate the SLC52A2 expression in various cancer cell lines. The results demonstrated that the SLC52A2 was expressed in all 38 kinds of tumor cell lines. Specifically, the meningioma cell lines and colorectal cell lines express the highest levels of SLC52A2. The diffuse large B-cell lymphoma cell lines and T-cell acute lymphoblastic leukemia cell lines were the lowest (Additional file 4: Fig. S1B). 
Table 1 Full names and abbreviations of the 33 cancers in the TCGA database

\begin{tabular}{|c|c|}
\hline Abbreviation & Full name \\
\hline ACC & Adrenocortical carcinoma \\
\hline BLCA & Bladder urothelial carcinoma \\
\hline BRCA & Breast invasive carcinoma \\
\hline CESC & $\begin{array}{l}\text { Cervical squamous cell carcinoma and endocervi- } \\
\text { cal adenocarcinoma }\end{array}$ \\
\hline $\mathrm{CHOL}$ & Cholangiocarcinoma \\
\hline COAD & Colon adenocarcinoma \\
\hline DLBC & Lymphoid neoplasm diffuse large B-cell lymphoma \\
\hline ESCA & Esophageal carcinoma \\
\hline GBM & Glioblastoma multiforme \\
\hline HNSC & Head and neck squamous cell carcinoma \\
\hline $\mathrm{KICH}$ & Kidney chromophobe \\
\hline $\mathrm{KIRC}$ & Kidney renal clear cell carcinoma \\
\hline KIRP & Kidney renal papillary cell carcinoma \\
\hline LAML & Acute myeloid leukemia \\
\hline LGG & Brain lower grade glioma \\
\hline LIHC & Liver hepatocellular carcinoma \\
\hline LUAD & Lung adenocarcinoma \\
\hline LUSC & Lung squamous cell carcinoma \\
\hline MESO & Mesothelioma \\
\hline OV & Ovarian serous cystadenocarcinoma \\
\hline PAAD & Pancreatic adenocarcinoma \\
\hline PCPG & Pheochromocytoma and paraganglioma \\
\hline PRAD & Prostate adenocarcinoma \\
\hline READ & Rectum adenocarcinoma \\
\hline SARC & Sarcoma \\
\hline SKCM & Skin cutaneous melanoma \\
\hline STAD & Stomach adenocarcinoma \\
\hline TGCT & Testicular germ cell tumors \\
\hline THCA & Thyroid carcinoma \\
\hline THYM & Thymoma \\
\hline UCEC & Uterine corpus endometrial carcinoma \\
\hline UCS & Uterine carcinosarcoma \\
\hline UVM & Uveal melanoma \\
\hline
\end{tabular}

Next, we analyzed the difference in mRNA expression of SLC52A2 between various cancer and normal tissues using the ONCOMINE database. We found that the SLC52A2 expression was more elevated in all cancers (Fig. 1D). To further estimate SLC52A2 expression in multiple human cancers, we examined mRNA sequencing data from the TCGA database. After selecting tumors with the number of normal sample size $\geq 5$, we confirmed the above findings with a significant upregulation of SLC52A2 expression in all cancers compared to normal tissues, including BLCA, BRCA, CHOL, COAD, ESCA, GBM, HNSC, KICH, KIRC, KIRP, LUAD, LIHC, LUSC, PRAD, READ, STAD, THCA, and UCEC
(Fig. 1E). Because of the small number of normal tissues in the TCGA database, we further combined the GTEx database and the TCGA database by the pan-cancer platform SangerBox. In addition to confirming the above TCGA results, we also found that SLC52A2 was significantly highly expressed in ACC, CESC, LGG, OV, PAAD, SKCM, TCGT, and UCS, while significantly lowly expressed in LAML than normal tissues (Fig. 1F).

\section{Correlation analysis of SLC52A2 expression and the clinical features of multiple cancers}

We estimated the association between the SLC52A2 expression and the clinical features across human cancers using TCGA data. We found that SLC52A2 expression was significantly higher in grade 3-4 patients than in grade1-2 in CESC, KIRC, LGG, LIHC, UCEC (Fig. 2A). In ACC, BRCA, COAD, HNSC, KIRC, KIRP, LUSC, and THYM, we revealed that patients with stage III - IV expressed significantly higher than the patients with stage I-II (Fig. 2B). As for the race, we found that patients with the non-White population (Native Hawaiian or other Pacific Islander, Pacific Islander, Black or African American, American Indian or Alaska Native, and Asian) expressed significantly higher than the patients with the White population in BRCA, HNSC, KIRC, LIHC, and SKCM (Fig. 2C). We also found that the expression of SLC52A2 was significantly higher in males than in females in BRCA and LIHC (Fig. 2D). Finally, we analyzed the correlation between SLC52A2 expression and tumor status. This result shows that compared with tumor-free, SLC52A2 expression in the presence of tumors was higher in ACC, COAD, KIRC, KIRP, LGG, PRAD, READ, THYM, and UVM (Fig. 2E). Thorsson et al.[18]. found six stable and reproducible immune subtypes, including wound healing (C1), IFN- $\gamma$ dominant $(\mathrm{C} 2)$, inflammatory (C3), lymphocyte deplete (C4), immunologically quiet (C5), and TGF- $\beta$ dominant (C6), which cover almost all cancers. These subtypes were related to prognosis, genetic, and immune-modulatory alterations that may shape the specific types of immune environments. Here, we examined the expression of SLC52A2 in different immune subtypes in multiple cancers and found a significant difference in BRCA, KIRC, $\mathrm{KICH}, \mathrm{TGCT}$, LIHC, LUAD, LUSC, OV, PAAD, PRAD, SARC, and STAD (Additional file 5: Fig. S2). Interestingly, SLC52A2 expression was lowest in the C3 immune subtype and higher in the $\mathrm{C} 4$ immune subtype.

Prognostic analysis of SLC52A2 in multiple human cancers TCGA database was used to estimate the correlation between SLC52A2 expression and survival in multiple human cancers. When the median SLC52A2 expression value of 33 types of human cancers was chosen as the 


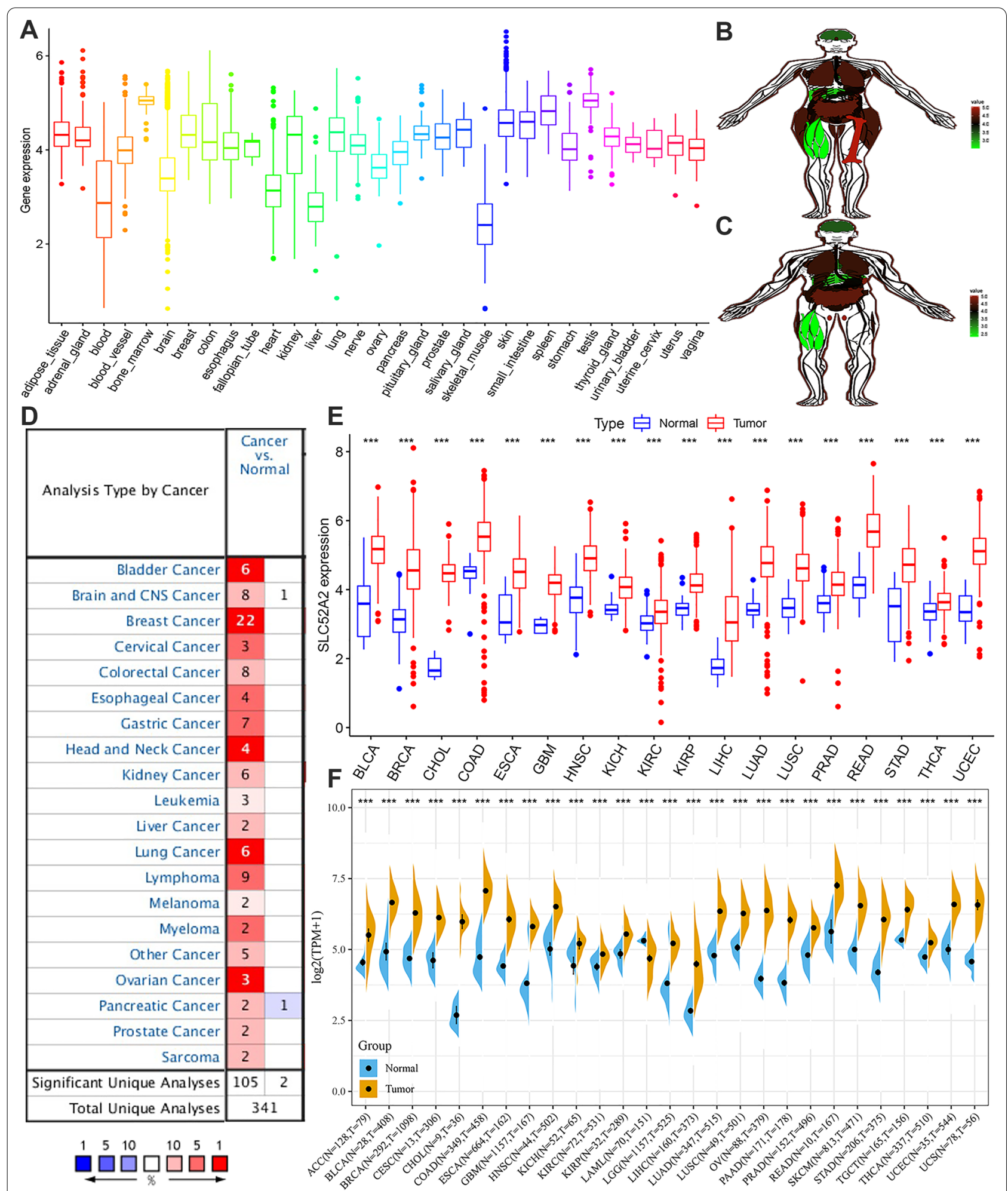

Fig. 1 mRNA expression of SLC52A2 gene in various cancers and its corresponding normal tissues. A Normal tissues expression. B Female expression anatomy diagram. C Male expression anatomy diagram. D Oncomine dataset. E TCGA dataset. F TCGA and GTEx dataset (***P<0.001) 


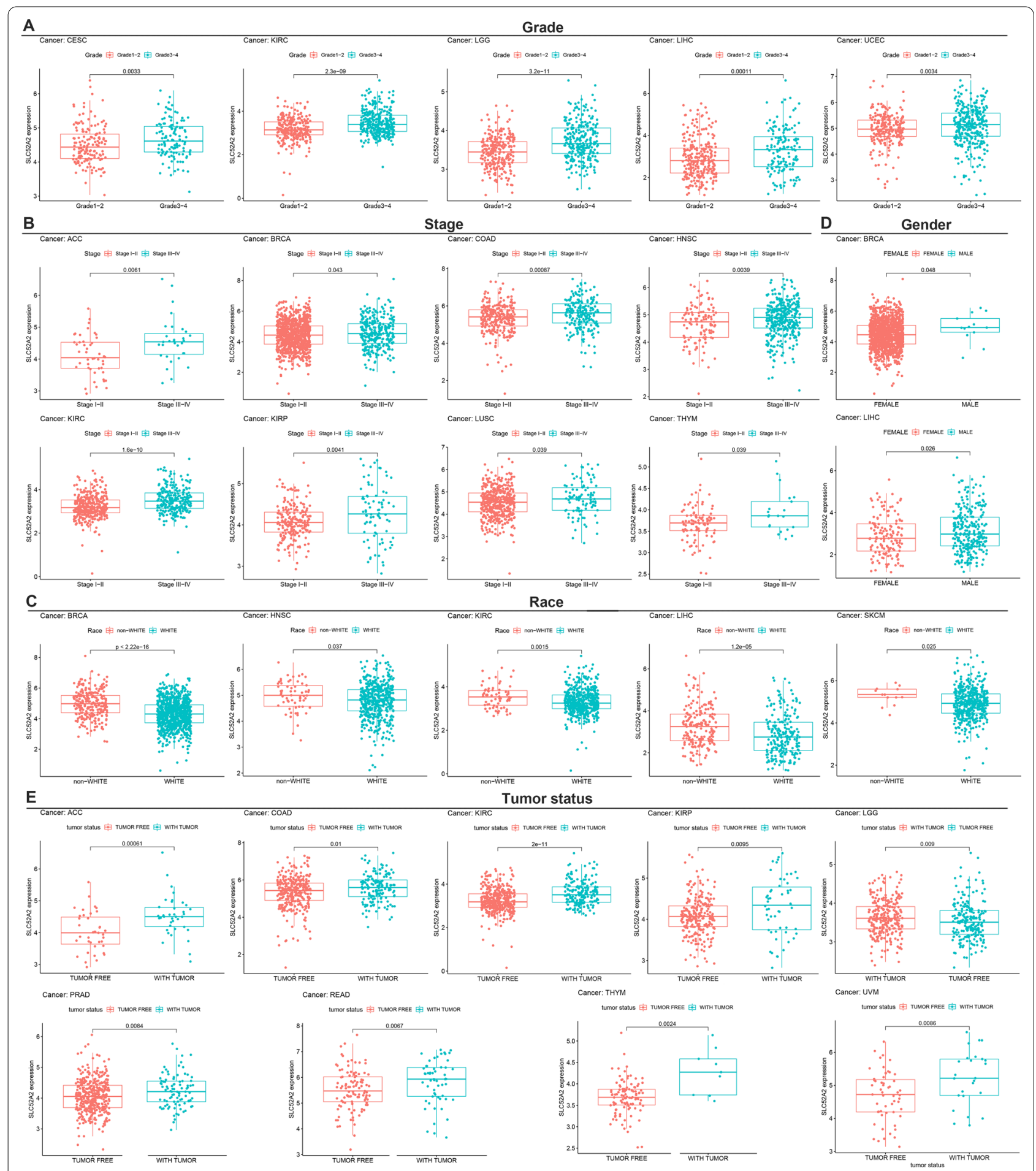

Fig. 2 Correlation of SLC52A2 mRNA expression levels with clinical characteristics. Correlation between SLCC52A2 and grade (A), stage (B), race (C), gender (D), and tumor status (E)

cut-off value by Kaplan-Meier analysis, the results have shown that the high expression of SLC52A2 was related to poor survival in ACC (OS, DSS, PFI), CESC (OS, PFI), HNSC (PFI), KIRC (OS, DSS, PFI), KIRP (OS, DSS), LGG
(OS, DSS, PFI), LIHC (OS), SARC (DSS), MESO (OS) and UVM (OS, DSS, PFI), whereas no statistical differences existed in other tumors (Fig. 3A). 
We further used Cox analysis to explore the SLC52A2-related survival (OS, DSS, and PFI) (Fig. 3BD). We thus identified that SLC52A2 acted as a detrimental prognostic factor in ACC (OS, DSS, PFI), CESC (OS, PFI), GBM (OS, DSS), KIRC (OS, DSS, PFI), KIRP (OS, DSS, PFI), LGG (OS, DSS, PFI), LIHC (OS, DSS), MESO (OS, DSS, PFI), PRAD (PFI), SARC (OS, DSS), SKCM (PFI), THYM (DSS), UCS (PFI) and UVM (OS, DSS, PF). While SLC52A2 expression was a protective prognostic factor in PCPG (DSS) (Fig. 3C).

Finally, we also explored the diagnostic value of SLC52A2 expression in pan-cancer. We selected cancers from the TCGA database with the number of normal controls in more than 40 samples and calculated the ROC curves and AUC. The AUC of the ROC had values higher than 0.70 in all analyses, including BRCA (AUC $=0.943), \quad \mathrm{COAD} \quad(\mathrm{AUC}=0.913)$, HNSC $(A U C=0.941), \quad$ KIRC $(A U C=0.706), \quad$ LIHC $(\mathrm{AUC}=0.954), \quad$ LUAD $\quad(\mathrm{AUC}=0.954), \quad$ LUSC $(\mathrm{AUC}=0.966), \quad$ PRAD $\quad(\mathrm{AUC}=0.794)$, and THCA $($ AUC $=0.721)$ (Additional file 6: Fig. S3), indicating a reasonable predictive model performance.

\section{SLC52A2 mutations in multiple human cancers}

We analyzed the alteration frequency of SLC52A2 in pan-cancer by the cBioPortal database. The results indicated that SLC52A2 was altered in 691 of the 10,953 patients (6\%) (Additional file 7: Fig. S4A). The highest alteration frequency was associated with amplification, followed by deep deletion and mutation. Among pan-cancer, OV had the highest alteration ratio with $26.2 \%$. Additional file 7: Fig. S4B further shows the type, sites, and case numbers of mutations in the SLC52A2 gene. 72 mutation sites (including 58 missense, 9 truncating, 4 fusion, and 1 inframe) located between amino acids 0 and 445 were identified in SLC52A2. We further analyzed the mutation distribution of the SLC52A2 gene in multiple human cancers by the COSMIC database. The results showed that missense mutations and synergistic mutations were the main mutation types in cancers (Additional file 7: Fig. S4C). The $\mathrm{C}>\mathrm{T}$ and $\mathrm{G}>\mathrm{A}$ mutations were most common in the SLC52A2 coding chain, and A $>G$ and $\mathrm{G}>\mathrm{T}$ mutations were also common (Additional file 7: Fig. S4C). Besides, we estimated the correlation between genetic alteration of SLC52A2 and the prognosis of cancers by the cBioPortal database. The data of Additional file 7: Fig. S4D-G indicate that cancer patients with altered levels of SLC52A2 showed poor prognosis in the progress-free interval and diseasefree interval compared with cases without SLC52A2 alteration.
Association of SLC52A2 expression with MSI, TMB and some common immune checkpoint genes in multiple human cancers

Currently, MSI and TMB status are important indicators of prognosis for patients with tumors and can facilitate the choice of treatment, especially the application of immune checkpoint inhibitors (ICIs) [19]. Therefore, we estimated the relationship between the MSI, TMB, and SLC52A2 expression in pan-cancer. Our results revealed that SLC52A2 expression was positively associated with MSI in THCA, STAD, SKCM, LUSC, KIRC, $\mathrm{KICH}, \mathrm{HNSC}$, ESCA, DLBC, and CESC, but negatively associated with MSI in READ and COAD (Fig. 4A). As for TMB, our results also demonstrated that the SLC52A2 expression was significantly related to TMB in 13 human cancers. Among these, the expression of SLC52A2 had a positive association with TMB in 12 cancer types, including ACC, STAD, SARC, PRAD, PAAD, LUSC, LUAD, LGG, KIRC, ESCA, BRCA, BLCA. In contrast, the SLC52A2 expression had a negative association with TMB in COAD (Fig. 4B). Besides, we also used the mRNA sequence data from the TCGA database to assess whether there is an association between SLC52A2 expression and some common immune checkpoint genes. The result revealed that the significant coexpression of SLC52A2 and most immune checkpoint genes were detected in KICH, KIRC, KIRP, LGG, LIHC, and UVM (Fig. 4C).

\section{Association between SLC52A2 expression with immune cells infiltration in multiple human cancers}

It is well known that immune cells play a very important role in the immune microenvironment and can influence the prognosis of cancer patients. Here, we further analyzed the correlation between SLC52A2 expression and infiltration of 6 immune-related cells by the TIMER database. The results showed that SLC52A2 was positively correlated with most immune cell infiltration in KIRP, LGG, and LIHC (Fig. 4D). We further explored the relationship of SLC52A2 with different functional immune infiltrating cells, including $\mathrm{T}$ cell (general, $\mathrm{CD}^{+}$, Th1, Th2, Tfh, Th17, Treg, and $\mathrm{T}$ cell exhaustion), B cell, monocyte, tumor-assisting macrophages (TAMs), M1 and M2 macrophage, neutrophil, and Dendritic cell. As shown in Fig. 4E, we were excited to observe a strong positive correlation between SLC52A2 and the molecular markers of T cell exhaustion in BLCA, KICH, KIRC, KIRP, LGG, LIHC, PCPG, SARC, TCGT, THCA, THYM, and UVM. Besides, the SLC52A2 and molecular markers of TAMs and M2 macrophages also showed a significant positive correlation in KICH, LIHC, SARC, and UVM. Thus, SLC52A2 


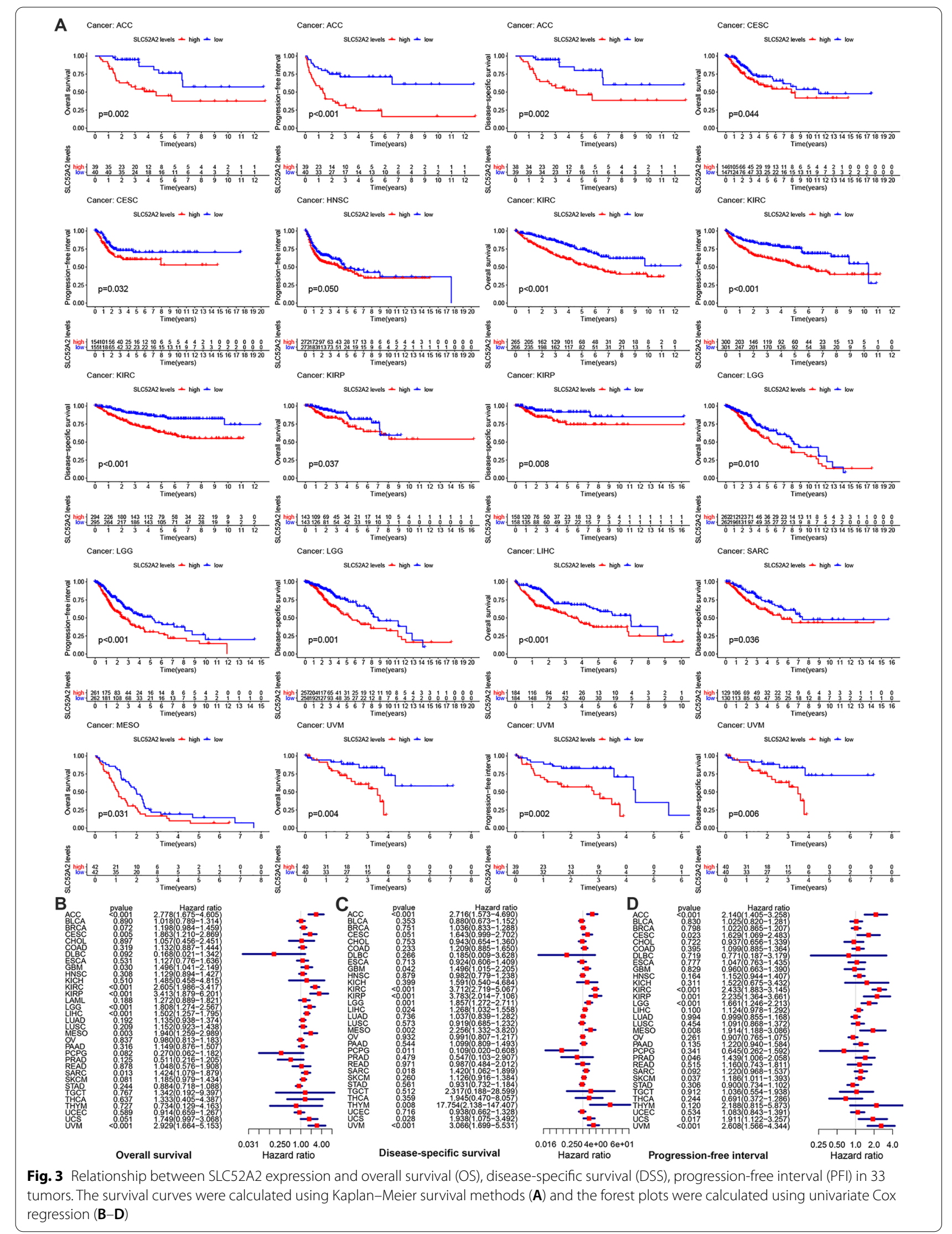




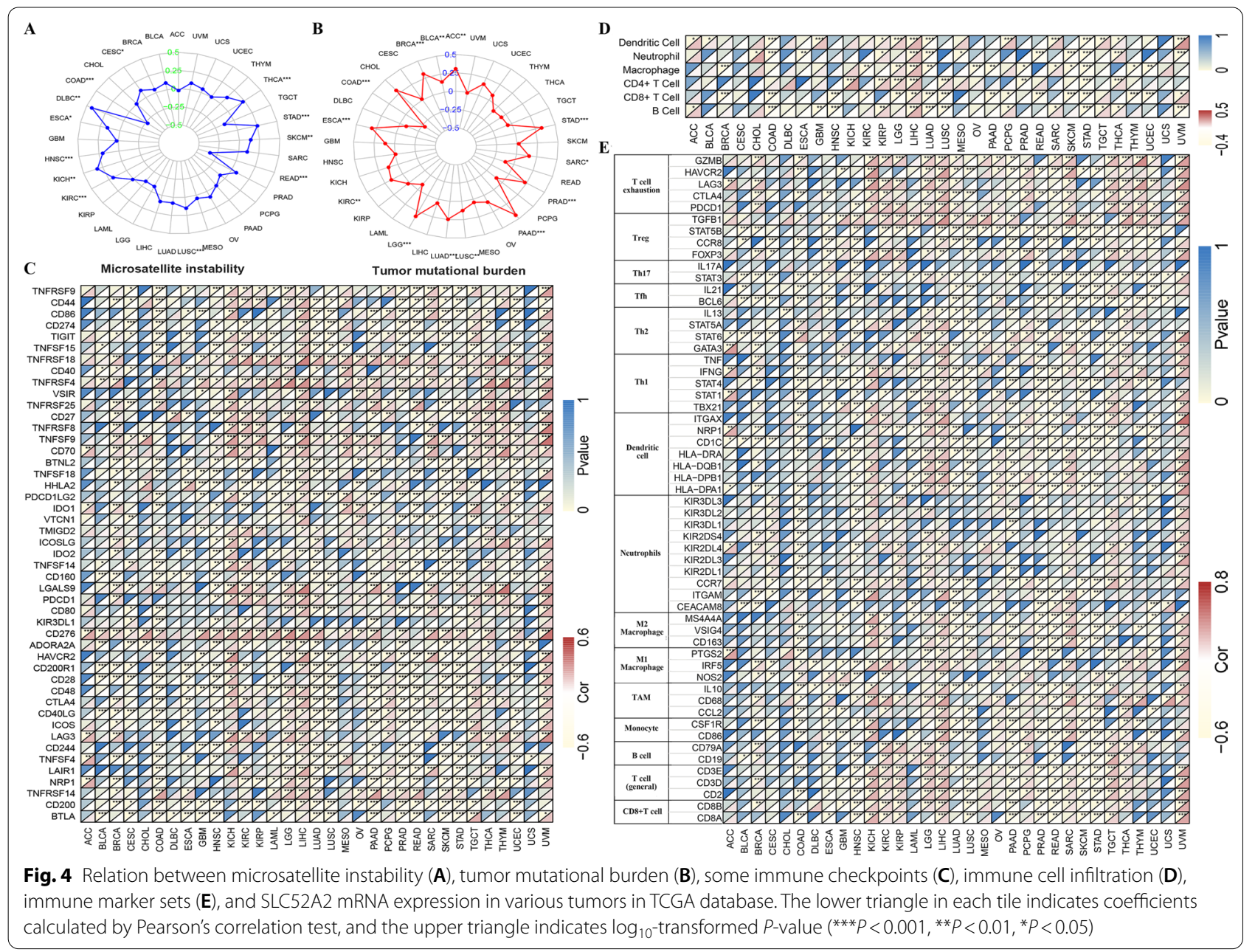

may play a crucial role in immune escape and macrophage M2 polarization in the cancers microenvironment. In addition, the correlation between SLC52A2 expression and infiltration of 22 immune cells was analyzed in a variety of tumors according to the CIBERSORT algorithm. Our results are roughly consistent with the above findings (Additional file 8: Fig. S5). For example, the results show that SLC52A2 is positively related to the level of $\mathrm{M} 2$ macrophage infiltration in HNSC, LGG, LIHC, SARC, and THYM. We also found that the level of Tregs infiltration, which contributes to immune escape, positively correlated with SLC52A2 expression in BRCA, KIRC, KIRP, LGG, LIHC, LUAD, PAAD, PCPG, PRAD, and STAD (Additional file 8: Fig. S5). It is worth noting that, using the TIDE algorithm, we found that in most tumors, the infiltration level of myeloid-derived suppressor cells is positively associated with the expression of SLC52A2 (Additional file 8: Fig. S5). In summary, we found that SLC52A2 is closely related to the formation of the cancer-promoting immune microenvironment.

\section{SLC52A2-related gene enrichment analysis}

To further explore the role of SLC52A2 in tumorigenesis, we used the GEPIA2 tool to obtain 114 genes that were highly correlated with SLC52A2 expression $(R>0.4$, Additional file 2: Table S2). Then, the protein interaction network of the above genes was built using the STRING tool (Fig. 5A). We further perform KEGG and GO enrichment analyses. After the screening of GO enrichment analysis, we revealed several enriched gene sets shown in Fig. 5B. The biological process (BP) is mainly enriched in "ribonucleoprotein complex biogenesis", "ncRNA processing", "ncRNA metabolic process" and "ribosome biogenesis". For the result of the cellular component (CC), it was indicated that these genes were mainly related to "preribosome, large subunit precursor" and "preribosome". Moreover, in the molecular function (MF) analysis, "intramolecular transferase activity" was found to be related to these genes. The KEGG data of Fig. 5B show that "Oocyte meiosis", "Ribosome biogenesis in eukaryotes", "Cell cycle" and "Ribosome" might be involved in the effect of SLC52A2 on tumor pathogenesis. 

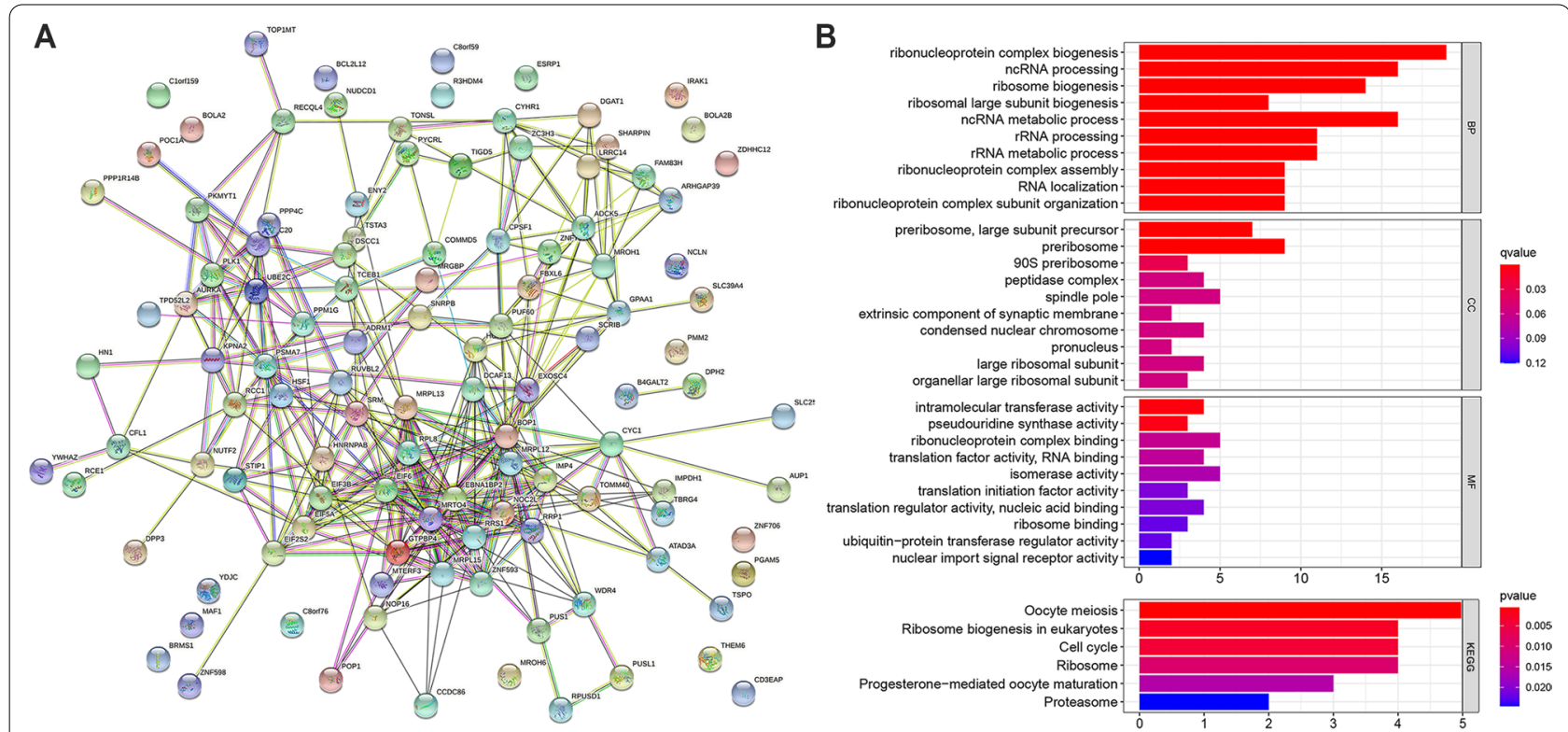

Fig. 5 PPI network analysis and KEGG and GO enrichment analysis of SLC52A2-related genes. A The PPI network of SLC52A2 is constructed by the GEPIA2 tool and STRING database. B The barplot of GO enrichment analysis and KEGG enrichment analysis. KEGG Kyoto Encyclopedia of Genes and Genomes, GO Gene Ontology, BP biological process, CC cellular component, MF molecular function

\section{Analysis of the relationship between SLC52A2 expression and LIHC}

From the above analysis, it was found that SLC52A2 was closely related to the prognosis of LIHC. Therefore, we conducted a more in-depth study on the correlation between SLC52A2 and LIHC. We first performed a pairwise difference analysis of LIHC using the TCGA database, and the results continued to show that SLC52A2 expression was much higher in tumors than in paracancerous tissues (Additional file 9: Fig. S6). Next, we validated the expression of SLC52A2 using the HCCDB database (including GSE22058, GSE25097, GSE36376, GSE14520, GSE54236, GSE63898, GSE64041, GSE76427, and ICGC-LIRI-JP datasets), GSE121248, GSE112790, GSE39791, GSE55092, GSE62232, and GSE102079 datasets, and results showed that SLC52A2 was highly expressed in LIHC (Fig. 6A-G). We also verified SLC52A2 mRNA expression by qRT-PCR analysis using hepatocellular carcinoma and paraneoplastic frozen tissues, and the results were consistent with the above (Fig. 6H). Finally, we further evaluated the SLC52A2 expression using IHC. IHC staining showed that SLC52A2 was significantly upregulated in LIHC (Fig. 6I, M; Table 2). Besides, we obtained similar findings in STAD, READ, and COAD (Fig. 6J-M; Additional file 3: Table S3). Based on IHC analysis, 40 patients were divided into a low SLC52A2 expression group $(\mathrm{n}=20)$ and a high SLC52A2 expression group $(\mathrm{n}=20)$. Detailed clinicopathological characteristics in LIHC are summarised in Table 2. Chi-squared analysis revealed that male patients $(P=0.034)$ and patients with larger tumor size $(P=0.043)$, poor tumor differentiation $(P=0.025)$, TNM stage III-IV $(P=0.157)$, vascular invasion $(P=0.236)$ appeared to exhibit the higher expression of SLC52A2.

We also validated the relationship between SLC52A2 and the prognosis of LIHC patients using the GSE14520 dataset, which showed that high SLC52A2 expression in tumor tissues correlated with poor prognosis of patients (Fig. 7A, B). Therefore, we further explored whether SLC52A2 is an independent prognostic factor for LIHC patients using TCGA data. As seen in Fig. 7C, D, the SLC52A2 expression was significantly associated with OS in LIHC in the univariate and multivariate Cox regression, indicating SLC52A2 expression was an independent predictor of prognosis in LIHC. To establish a clinically applicable method that could predict the survival probability of patients with LIHC, we conducted nomogram predictive models [C-index: 0.655(0.597-1), $P<0.001$ ], considering SLC52A2 expression and clinicopathological features, to predict the probability of the 1-, 2-, 3- and 5-year OS (Fig. 7E). The calibration plots for the 1-, 2and 3-year OS rates were predicted well compared with an ideal model (Fig. 7F). In addition to LIHC, we found SLC52A2 to be an independent risk factor in CESC, KIRC, and KIRP (Additional file 10: Fig. S7).

Finally, to further validate the reliability of the above SLC52A2 enrichment analysis results and to further 


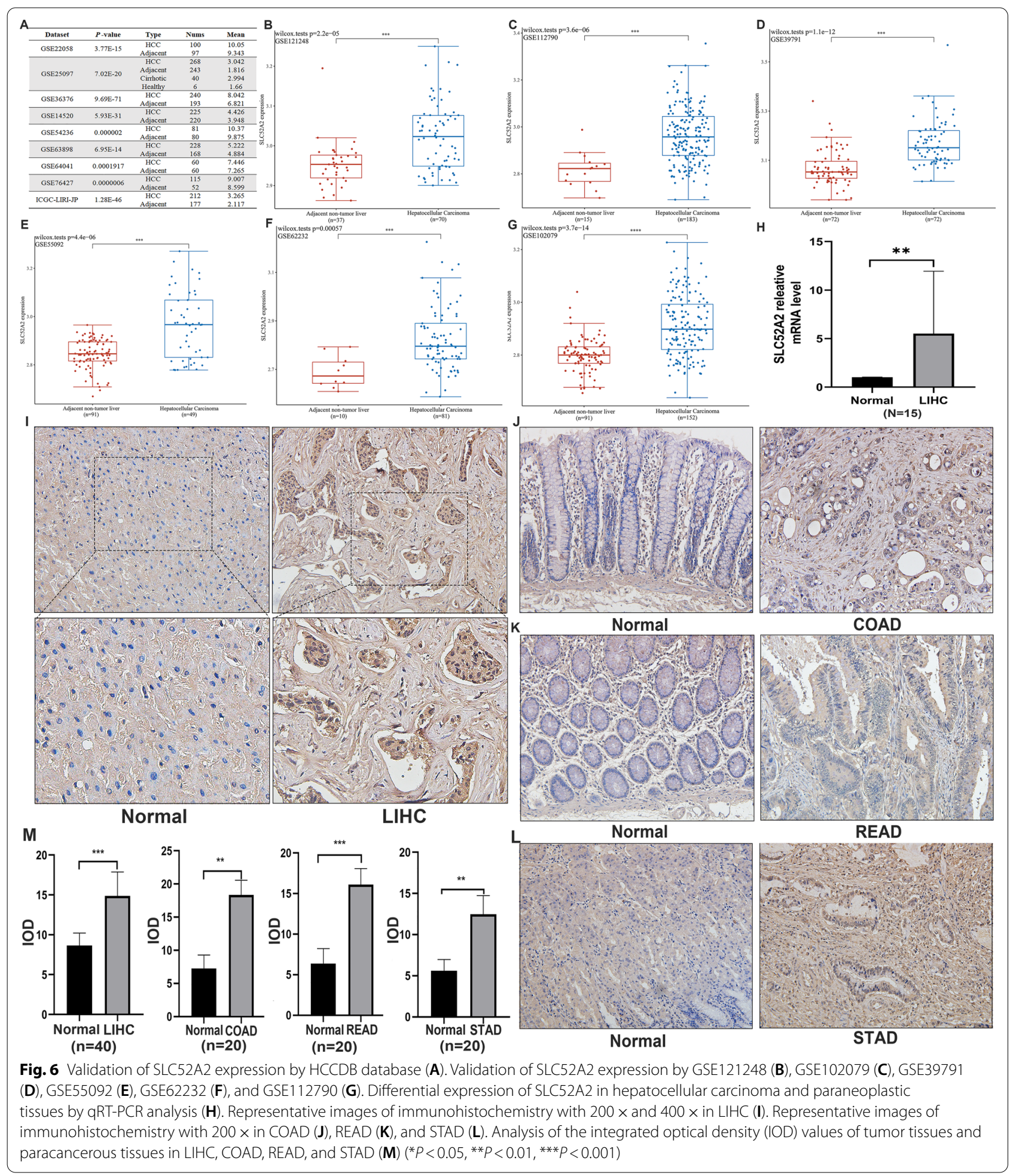

explore the role of SLC52A2 in the progression of LIHC, we divided the patients into high and low expression groups according to their median SLC52A2 expression levels and performed KEGG and GO enrichment analysis. The results were consistent with the above analysis (Additional file 11: Fig. S8). For example, compared with patients with low SLC52A2 expression, patients with high SLC52A2 expression are significantly enriched 
Table 2 The association between SLC52A2 expression and clincopathological features in LIHC

\begin{tabular}{|c|c|c|c|c|}
\hline \multirow{2}{*}{$\begin{array}{l}\text { Clinical } \\
\text { variables }\end{array}$} & \multirow{2}{*}{$\begin{array}{l}\text { No. of } \\
\text { patients } \\
n=40\end{array}$} & \multicolumn{2}{|c|}{ SLC52A2 expression level } & \multirow[t]{2}{*}{$P$ value } \\
\hline & & $\operatorname{Low}(n=20)$ & High $(n=20)$ & \\
\hline \multicolumn{5}{|l|}{ Gender } \\
\hline Male & 29 & 11 & 18 & 0.034 \\
\hline Female & 11 & 9 & 2 & \\
\hline \multicolumn{5}{|l|}{ Age (years) } \\
\hline$<60$ & 31 & 18 & 13 & 0.130 \\
\hline$\geq 60$ & 9 & 2 & 7 & \\
\hline \multicolumn{5}{|l|}{$\mathrm{HBsAg}$} \\
\hline Positive & 36 & 18 & 18 & 1 \\
\hline Negative & 4 & 2 & 2 & \\
\hline \multicolumn{5}{|l|}{$\operatorname{AFP}(n g / m l)$} \\
\hline$<400$ & 16 & 10 & 6 & 0.197 \\
\hline$\geq 400$ & 24 & 10 & 14 & \\
\hline \multicolumn{5}{|l|}{ Liver cirrhosis } \\
\hline Yes & 28 & 13 & 15 & 0.490 \\
\hline No & 12 & 7 & 5 & \\
\hline \multicolumn{5}{|l|}{$\begin{array}{l}\text { Child-Pugh } \\
\text { Class }\end{array}$} \\
\hline$A$ & 33 & 18 & 15 & 0.405 \\
\hline B & 7 & 2 & 5 & \\
\hline \multicolumn{5}{|l|}{$\begin{array}{l}\text { Tumor size } \\
(\mathrm{cm})\end{array}$} \\
\hline$<5 \mathrm{~cm}$ & 13 & 10 & 3 & 0.043 \\
\hline$\geq 5 \mathrm{~cm}$ & 27 & 10 & 17 & \\
\hline \multicolumn{5}{|c|}{$\begin{array}{l}\text { Vascular inva- } \\
\text { sion }\end{array}$} \\
\hline Yes & 8 & 2 & 6 & 0.236 \\
\hline No & 32 & 18 & 14 & \\
\hline \multicolumn{5}{|l|}{$\begin{array}{l}\text { Tumor dif- } \\
\text { ferentiation }\end{array}$} \\
\hline Well & 23 & 15 & 8 & 0.025 \\
\hline Poor & 17 & 5 & 12 & \\
\hline \multicolumn{5}{|l|}{ TNM stage } \\
\hline$|-| \mid$ & 29 & 17 & 12 & 0.157 \\
\hline III-IV & 11 & 3 & 8 & \\
\hline
\end{tabular}

in the "Oocyte meiosis", "Cell cycle" and "Ribosome" pathways (Additional file 11: Fig. S8).

\section{Prediction and analysis of upstream IncRNA and miRNAs of SLC52A2 in LIHC}

It is well known that non-coding RNA (ncRNA) is responsible for regulating gene expression. To determine whether SLC52A2 is regulated by certain ncRNAs, we first predicted upstream miRNAs that may bind to SLC52A2 using the starBase database and finally identified 13 miRNAs that were significantly associated (Additional file 12: Fig. S9A). However, based on the action mechanism of miRNAs in regulating target gene expression, there should be a negative association between miRNAs and SLC52A2. Therefore, we considered hsamiR-122-5p $(R=-0.473)$ and hsa-miR-139-5p $(R=$ - 0.449), which have the largest negative correlation coefficients, maybe the upstream miRNA of SLC52A2. As shown in Fig. 8A, B, hsa-miR-139-5p was expressed at much lower levels in tumors, and low expression was closely associated with poor prognosis. Figure $8 \mathrm{C}$ shows a match analysis of the SLC52A2 sequences and has-miR139-5p sequences using the StarBase database. Although the expression of hsa-miR-122-5p was also lower in cancer, and prognostic analysis found that its high expression was associated with poor prognosis (Additional file 12: Fig. S9B, C), which contradicted the relationship between SLC52A2 and prognosis. Thus, we speculate that hsa-miR-139-5p is the most likely upstream miRNA of SLC52A2.

Next, the starBase database was used to predict the upstream lncRNAs of hsa-miR-139-5p. A total of 29 possible lncRNAs were predicted (Additional file 12: Fig. S9D). The results showed that the largest negative correlation coefficients with hsa-miR-139-5p were for SNHG3 $(R=-0.443)$ and THUMPD3-AS1 $(R=-0.362)$. Based on the competing endogenous RNA (ceRNA) hypothesis, lncRNA could rise the expression of mRNA by competitively binding to shared miRNAs. Thus, there should be a positive correlation between IncRNA and mRNA or a negative relationship between lncRNA and miRNA. As shown in Fig. 8D-I, we found that SNHG3 and THUMPD3-AS1 were more highly expressed in LIHC and were associated with poor prognosis by GEPIA2. Besides, we also found that SLC52A2 expression was positively correlated with SNHG3 $(R=0.559)$ and THUMPD3-AS1 $(R=0.437)$ using the starBase database (Fig. 8J, K). Taking expression analysis, survival analysis, and correlation analysis into consideration, SNHG3 and THUMPD3-AS1 might be the two most potential upstream lncRNAs of the hsa-miR-139-5p-SLC52A2 axis in LIHC.

\section{Discussion}

Despite the close association of SLC52A2 with human health, little data is available on this transporter, especially in tumors. In this study, we systematically analyzed the association of SLC52A2 with multiple human tumor types. We found that of the 28 tumors whose normal tissues could acquire through the ONCOMINE, GTEx, and TCGA databases, 27 of them were highly expressed, and SLC52A2 expression increased with increasing tumor stage in multiple tumors. As previous studies have demonstrated that riboflavin in the blood of tumor patients is reduced $[20,21]$. Thus, we hypothesize that the rapid 

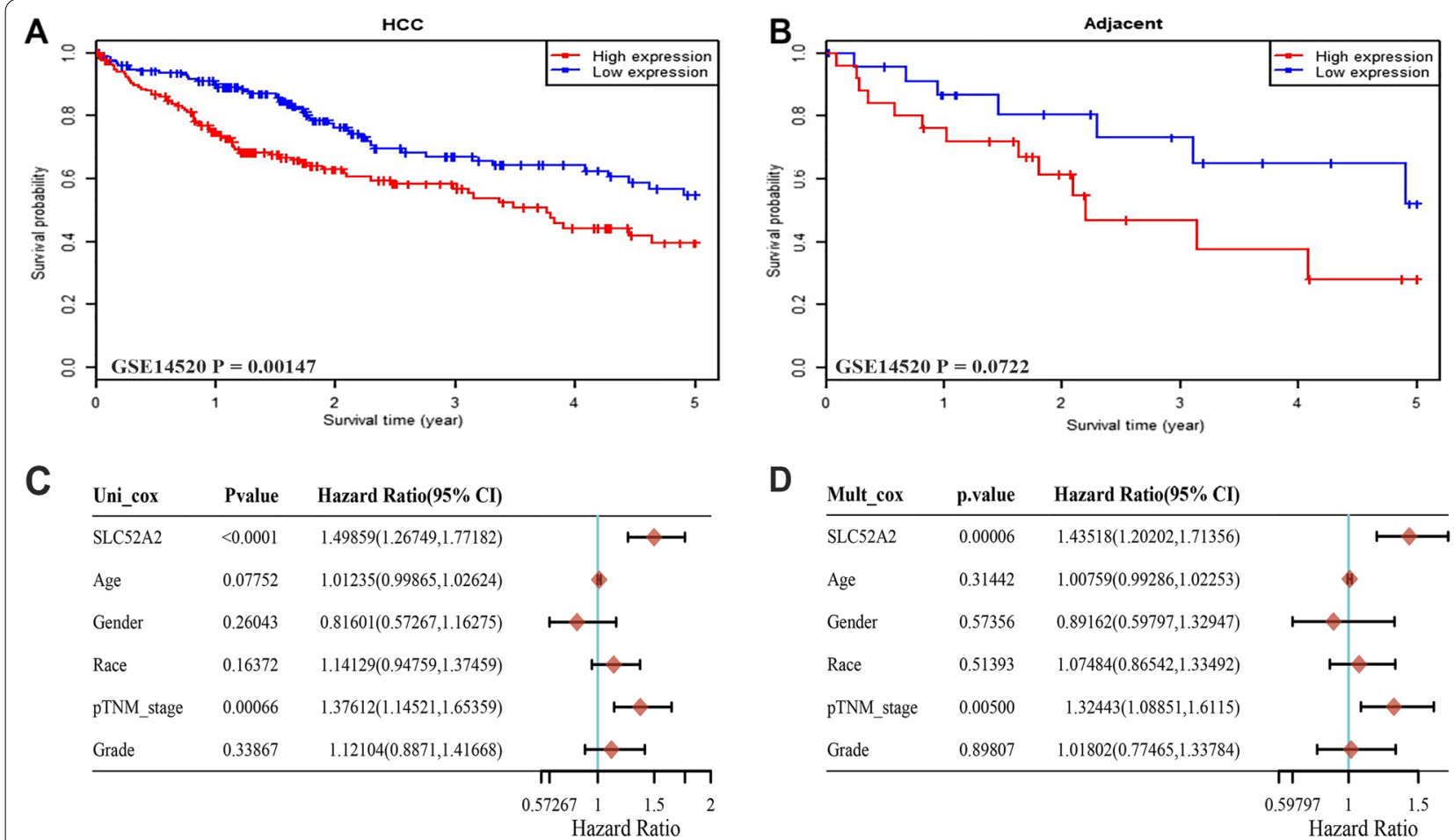

E
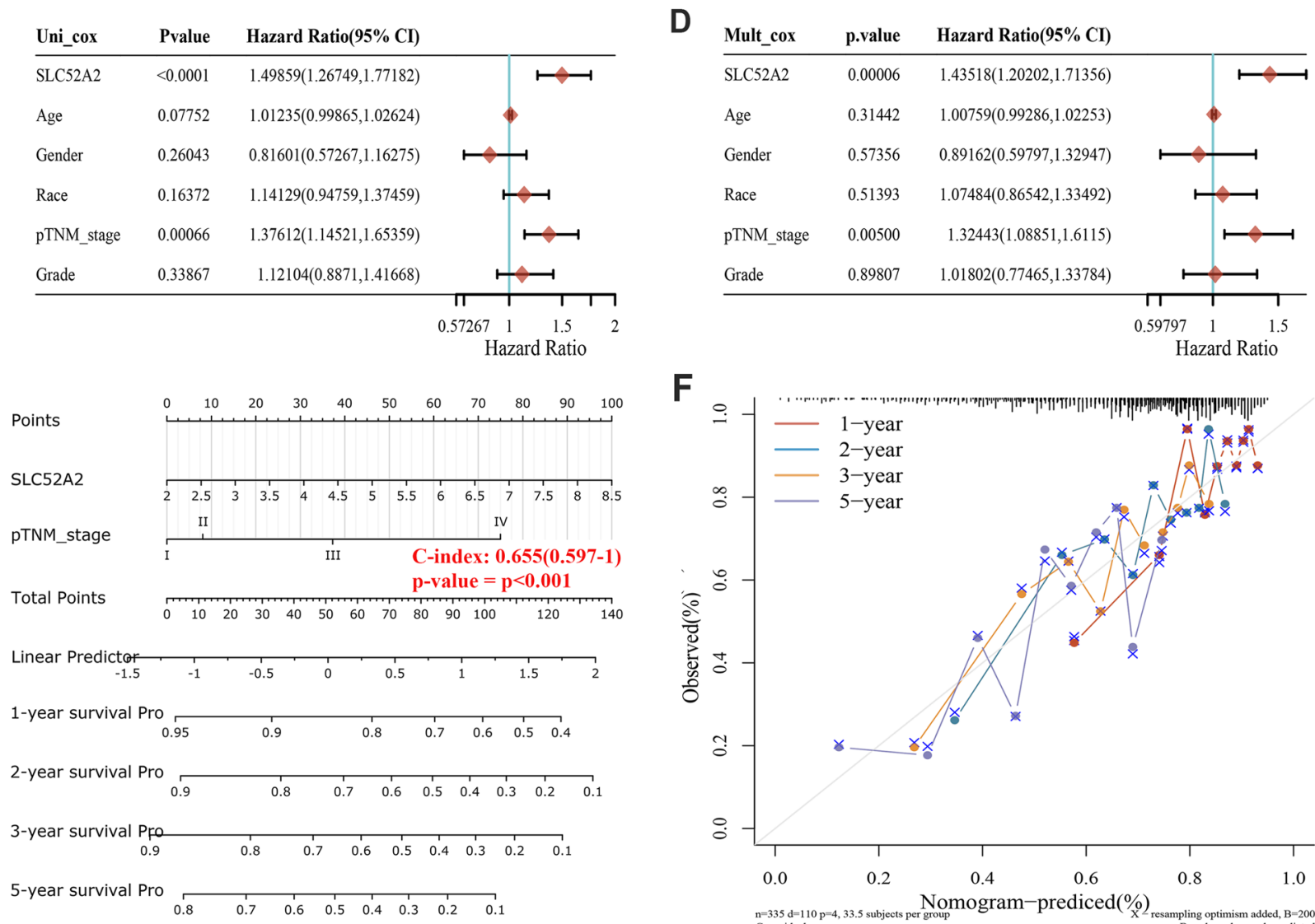

$\mathbf{F}$

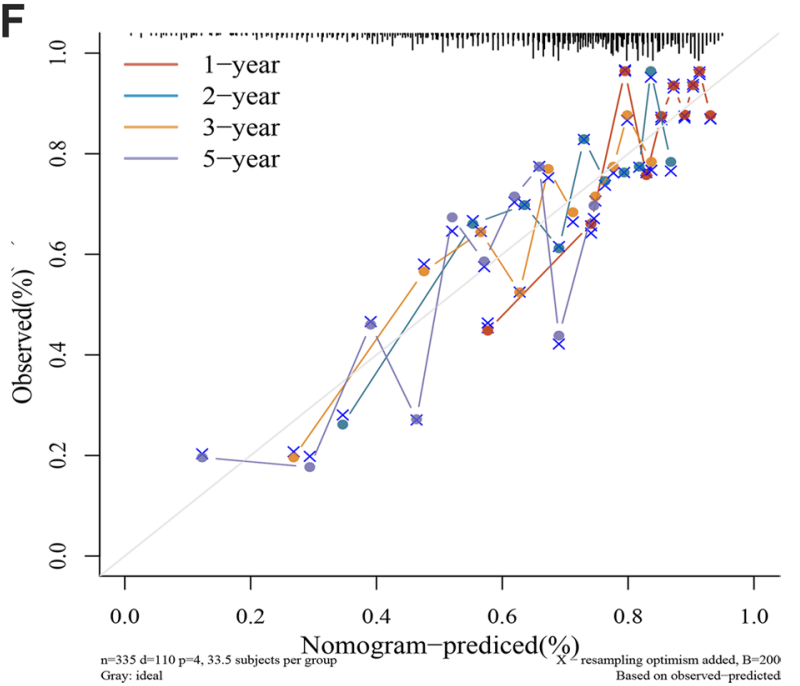

Fig. 7 Association between SLC52A2 expression and the prognosis in LIHC. GSE14520 dataset was used to verify the poor prognosis of SLC52A2 in LIHC patients (A, B). C, D Hazard ratio and P-value of constituents involved in univariate and multivariate Cox regression and some parameters of the SLC52A2 genes [age (years) is a continuous variable; Gender: Male vs Female (reference values); Race: Black/White vs Asian (reference values); pTNM stage: III/IV vs I/II (reference values); Grade: G3/G4 vs G1/G2 (reference values)]. E Nomogram to predict the 1-year, 2-year, 3-year, and 5-year overall survival of LIHC cancer patients. F Calibration curve for the overall survival nomogram model. A dashed diagonal line represents the ideal nomogram, and the red line, blue line, orange line, and purple line represent the 1-year, 2-year, 3-year, and 5-year observed nomograms

metabolism and proliferation of tumor cells increases the rate of riboflavin consumption and uptake, which in turn induces the SLC52A2 expression in the organism to facilitate the supply of cytosolic riboflavin.

To explore whether the currently published articles on SLC52A2 expression and cancer are consistent with our conclusions, we searched PubMed, Embase, the Cochrane Library, on April 30, 2021, using the following terms in [All Fields]: BVVLS2, GPR172A, SLC52A2, HRFT3, GPCR41, RFVT2, RFT3, BVVLS2. We retrieved three relevant studies, and all were consistent with our findings from publicly available databases. For example, 


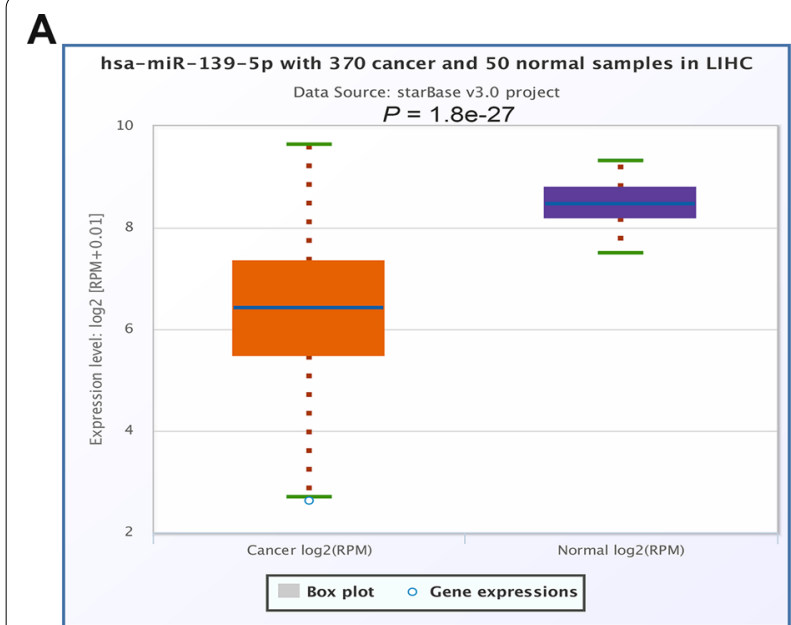

C

SLC52A2 5' gCCaGGGACCAGUGGGGGCUGUAGg 3'

$|:|||::||:||||||$

miR-139-5p $3^{\prime}$ ugacCUCUG--UGCACGUGACAUCu 5 '

$\mathbf{F}$

SNHG3 5' aUUGGAGUUUGGUGGACUGUAGa 3 '

|||| : ||| |||||||

miR-139-5p $3^{\prime}$ ugACCUCUGUGCACGUGACAUCu $5^{\prime}$

G

THUMPD3-AS15' ugguuacuuuaaaUACUGUAGu 3'

: || |||||

miR-139-5p $3^{\prime}$ ugaccucugugcacGUGACAUCu 5 '
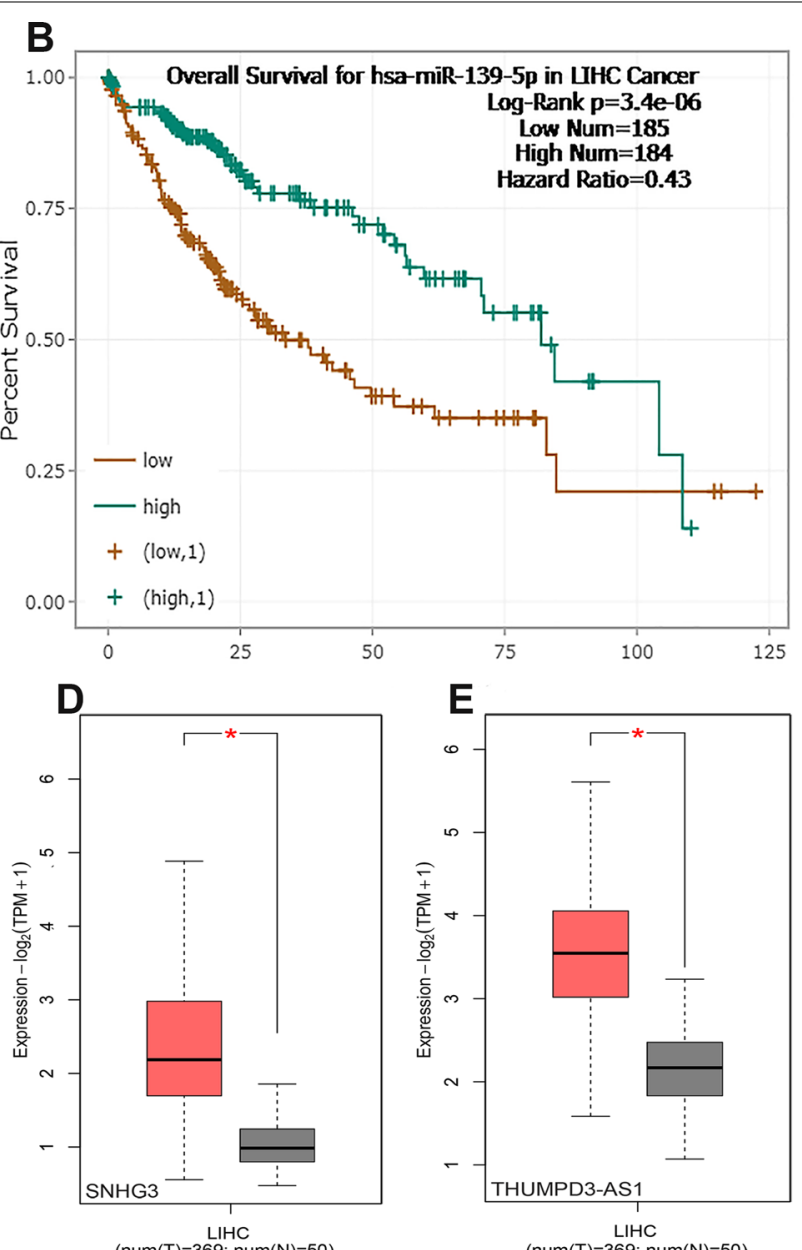

$(\operatorname{num}(\mathrm{T})=369 ; \operatorname{inum}(\mathrm{N})=50)$

$(\operatorname{num}(\mathrm{T})=369 ; \operatorname{num}(\mathrm{N})=50)$
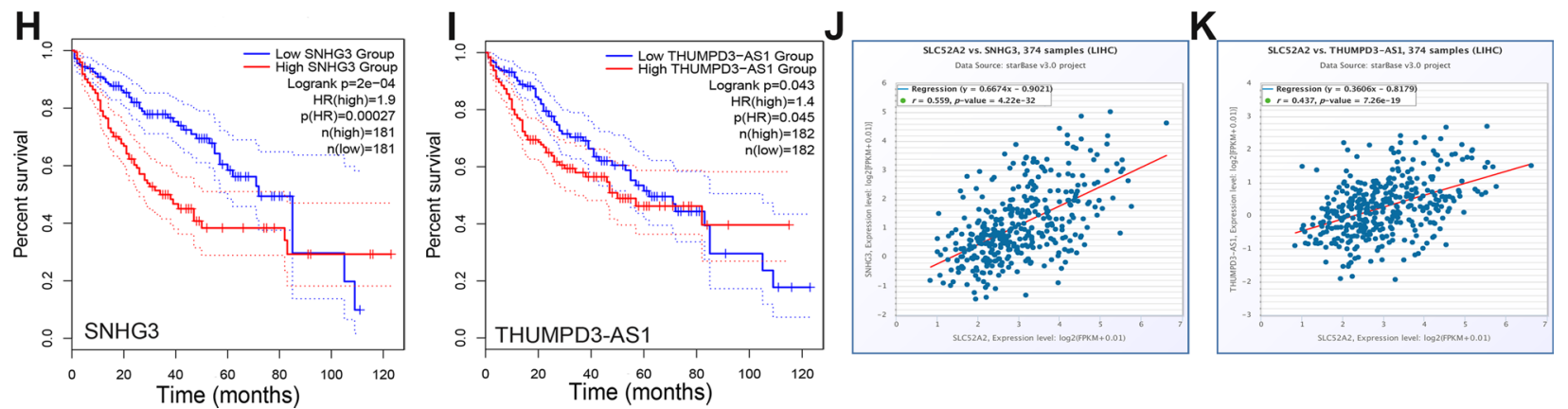

Fig. 8 Prediction and analysis of upstream IncRNA and miRNAs of SLC52A2 in LIHC. A Analysis of hsa-miR-139-5p expression in hepatocellular carcinoma and paraneoplastic tissues using starbase database. B Correlation of hsa-miR-139-5p with prognosis using the starbase database. C The matching analysis of related sequences of SLC52A2 and has-miR-139-5p. D, E Analysis of SNHG3 and THUMPD3-AS1 expression in hepatocellular carcinoma and paraneoplastic tissues using the GEPIA2. F, G The matching analysis of related sequences of SNHG3, THUMPD3-AS1, and has-miR-139-5p. H, I Correlation of SNHG3 and THUMPD3-AS1 with prognosis using the GEPIA2. J, K The SLC52A2 and SNHG3, THUMPD3-AS1 co-expression analysis using the starbase database $\left({ }^{*} P<0.05\right)$

by analyzing 80 paired gastric cancer and corresponding adjacent non-cancerous tissues in the GSE27342 dataset, Cheng et al. found that the SLC52A2 expression was elevated 1.7 -fold in gastric cancer, and the difference was statistically significant [10]. Similarly, Vathipadiekal found the significant upregulation of SLC52A2 in ovarian cancer tissues by using public data $(P<0.001)$. And, the above findings were then confirmed using the GSE26712 
dataset. Also, they compared serum SLC52A2 levels in patients with advanced ovarian cancer and normal age-matched controls by enzyme-linked immunosorbent assay. The results showed a 2.9 -fold increase in the patients with ovarian cancer [22]. Recently, Tutino et al. estimated the mRNA levels of SLC52A2 in tumor tissues and surrounding normal mucosa of 24 colorectal cancer patients. They found that SLC52A2 expression was increased in tumor tissues $(P<0.001)$. Notably, they also found that SLC52A2 protein levels were decreased in tumors as compared to normal mucosa, contrary to their results at the SLC52A2 mRNA level. We showed by immunohistochemical analysis that SLC52A2 was highly expressed in both COAD and READ [23].

We also found that high SLC52A2 expression was a risk factor and related to poor OS, DSS, and PFI. Interestingly, we also revealed that patients with cancer harboring SLC52A2 mutations had a worse prognosis. Besides, the SLC52A2 expression has good diagnostic value for multiple tumors. We then selected LIHC that were associated with all of these indicators for in-depth analysis, and we found that SLC52A2 expression was an independent prognostic indicator for patients with LIHC. Recently, it was found that the expression of SNHG3 was significantly higher in hepatocellular carcinoma than in paracancerous tissues [24]. and the overexpression of SNHG3 can promote cell invasion and epithelial-mesenchymal transition in liver cancer [25]. Wu et al. [26] revealed that hsa-miR-139-5p expression was reduced in liver cancer compared to normal liver tissues and that low miR-139-5p expression was associated with a poorer prognosis. Mechanistic studies suggested that low miR139-5p expression can increase the invasive and proliferative capacity of liver cancer cells. In the current study, we demonstrated that SNHG3 and THUMPD3-AS1/hsamiR-139-5p-SLC52A2 axis were identified as potential regulatory pathways in LIHC.

Immune checkpoints are a kind of suppressor molecule that acts as a beneficial effect in the human immune system, preventing inflammatory damage caused by the over-activation of $\mathrm{T}$ cells [27]. However, tumor cells can escape immune surveillance and achieve immuno-escape by overexpressing checkpoint molecules to suppress the anti-tumor response of the immune system [28, 29]. Our results show that SLC52A2 positively correlates with the expression of multiple immune checkpoint genes in pan-cancer, which is consistent with the conclusion that high SLC52A2 expression is related to a poor prognosis. The ICIs can prevent tumor progression by blocking the interaction of immune checkpoints and their ligands, disrupting immune tolerance, and promoting the clearance of tumor cells. A major advance in cancer therapy in recent years has been the development of ICIs, which have produced durable responses and improved survival in many kinds of solid malignancies [30-32]. However, the majority of patients treated with ICIs do not benefit and there is an urgent need to explore accurate predictive biomarkers of ICIs response to use them more selectively. TMB refers to the total number of somatic mutations per megabase of the interrogated genomic sequence, which varies across malignancies [33]. Increasing evidence indicates that the TMB may be a predictive biomarker for immunotherapy in a variety of solid tumors [34-36]. The higher the TMB of the cancer patients, the better the response to the ICI [37] and the longer the OS [38]. MSI was defined as any change in microsatellite length due to insertion or deletion of repetitive units in tumor tissues, with the emergence of new microsatellite DNA alleles. Similarly, MSI can also be used to guide decisions about adjuvant chemotherapy, targeted therapy, and immunotherapy for tumors $[39,40]$. Tumors with microsatellite instability-high were found to be sensitive to immune checkpoint blockade (ICB) [41]. Our study showed that the expression of SLC52A2 was positively associated with TBM and MSI in most tumor types. Therefore, tumor patients with high SLC52A2 expression may have a higher response rate to ICIs.

Immune infiltration in the tumor microenvironment plays a crucial role in tumor development and will affect the clinical prognosis of tumor patients [42]. We demonstrated that SLC52A2 is positively associated with most immune cell infiltration and immune marker sets of immune cells, such as TAMs, M2 macrophages, and T cell exhaustion in several tumors, including LIHC. As the most abundant innate immune cells in the tumor microenvironment, various studies have shown that TAMs can enhance tumorigenesis and progression by promoting angiogenesis in tumor tissues, strengthening the metastatic invasion of tumor cells, and suppressing anti-tumor immunity [43, 44]. As we know, M2 macrophages also have a role in promoting tumor growth, invasion, and metastasis $[45,46]$. Since TAMs possess most of the representative properties of M2 macrophages, TAMs are often considered to be M2 macrophages [47, 48]. T cell exhaustion refers to cancer patients where $\mathrm{T}$-cells are continuously provoked by antigens, cellular memory does not differentiate effectively and $\mathrm{T}$ cells gradually become exhausted. It is one of the main factors of immune dysfunction in cancer patients. Thus, SLC52A2 may be involved in the progression of tumors such as LIHC by potentially regulating $\mathrm{M} 2$ cell polarisation and inducing $\mathrm{T}$ cell depletion.

Finally, the KEGG and GO enrichment analysis disclosed that SLC52A2 was mainly enriched in oocyte meiosis, ribosome biogenesis in eukaryotes, and cell cycle. It is well known that the most important feature of cancer 


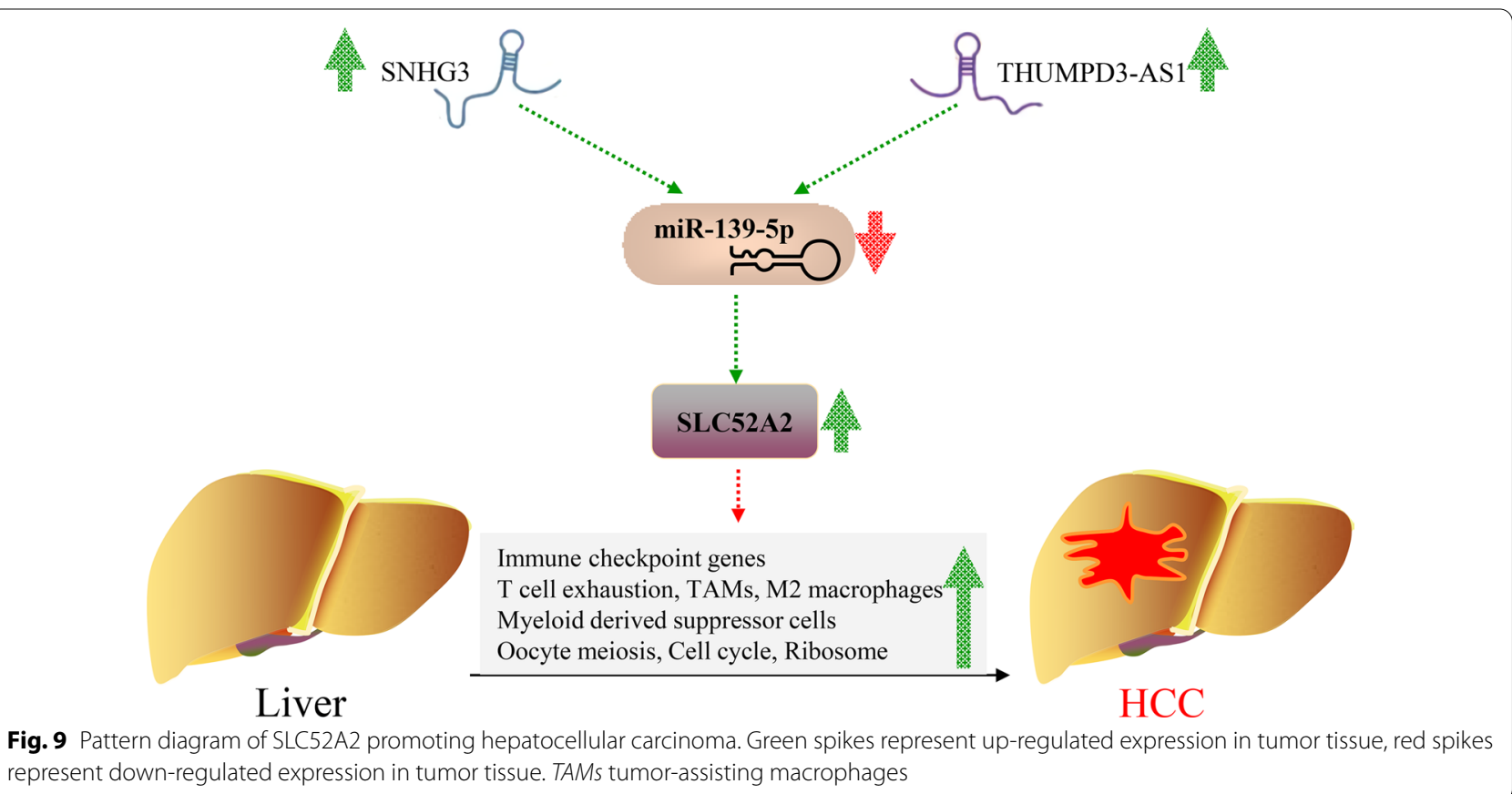

cells is their abnormal cell cycle activity and unlimited replication potential. Thus, the above pathway enriched by SLC52A2 may promote cancer progression.

Notably, although in this article we have conducted a comprehensive analysis of SLC52A2 in various tumors, there are still some limitations. This study is based on bioinformatics analysis and only validated the expression of SLC52A2 in LIHC, STAD, COAD, and READ at the tissue level, while the role of SLC52A2 in other tumors requires further experiments to confirm our results.

\section{Conclusion}

In conclusion, our study demonstrated that SLC52A2 was highly expressed in almost all tumors and associated with poor prognosis, diagnosis, mutations, TMB, MSI, common immune checkpoint genes, and immune cells infiltration in most cancers (Fig. 9). It may promote cancer progression through its involvement in oocyte meiosis, eukaryotic ribosome biogenesis, and cell cycle. Besides, the SLC52A2 expression was an independent prognostic factor for LIHC, and SNHG3 and THUMPD3-AS1/hsamiR-139-5p-SLC52A2 axis may be the potential regulatory pathways in LIHC (Fig. 9).

\section{Supplementary Information}

The online version contains supplementary material available at https://doi. org/10.1186/s12935-021-02432-7.

Additional file 1: Table S1. Database and its website.

Additional file 2: Table S2. 114 genes highly correlated with SLC52A2 expression using GEPIA2 $(R>0.4)$.

Additional file 3: Table S3. The clinicopathological details with STAD, COAD, and READ.

Additional file 4: Fig. S1. mRNA expression of SLC52A2 gene in normal human tissues (A). SLC52A2 mRNA expression in 38 kinds of tumor cell lines from the CCLE database (B). ( $\left.{ }^{* *} P<0.01\right)$.

Additional file 5: Fig. S2. SLC52A2 mRNA expression in different immune subtypes in BRCA, KIRC, KIRP, LGG, LIHC, LUAD, LUSC, OV, PAAD, PRAD, SARC, STAD, and TGCT.

Additional file 6: Fig. S3. The AUC of the ROC in BRCA, COAD, HNSC, KIRC, LIHC, LUAD, LUSC, PRAD, and THCA.

Additional file 7: Fig. S4. SLC52A2 mutation landscape. (A) SLC52A mutation level from the cBioPortal database. (B) Mutation diagram of SLC52A2 in different cancer types across protein domains from the cBioPortal database. (C) Pie chart showing the percentage of the different mutation types of SLC52A2 in cancers according to the COSMIC database. (D-G) The genetic alteration of SLC52A2 and the survival prognosis of cancers by the cBioPortal database.

Additional file 8: Fig. S5. the correlation between SLC52A2 expression and immune cell infiltration using CIBERSORT and TIDE algorithms.

Additional file 9: Fig. S6. Pairwise difference analysis of SLC52A2 expression in the TCGA-LIHC cohort. 
Additional file 10: Fig. S7. Hazard ratio and $P$-value of constituents involved in univariate and multivariate Cox regression and some parameters of the SLC52A2 genes in CESC(A), KIRC (B), and $\operatorname{KIRP}(C)$. [age (years) is a continuous variable; Gender: Male vs Female (reference values); Race: Black/White vs Asian (reference values); pTNM stage: III/IV vs I/II (reference values); Grade: G3/G4 vs G1/G2 (reference values)].

Additional file 11: Fig. S8. KEGG and GEO enrichment analysis in the LIHC-TCGA cohort. KEGG, Kyoto Encyclopedia of Genes and Genomes; GO, Gene Ontology; BP, biological process; CC, cellular component.

Additional file 12: Fig. S9. Prediction and analysis of upstream IncRNA and miRNAs of SLC52A2 in Hepatocellular carcinoma. (A) Correlation analysis of SLC52A2 and miRNA using starbase database. (B) Analysis of hsa-miR-122-5p expression in hepatocellular carcinoma and paraneoplastic tissue using starbase database. (C) Correlation of hsa-miR-122-5p with prognosis using the starbase database. (D) Correlation analysis of hsa-miR139-5p and IncRNA using starbase database.

\section{Acknowledgements}

None.

\section{Author's contributions}

$Z \mathrm{~L}$, and $\mathrm{LM}$ came up with the design and conception. $\mathrm{ZL}, \mathrm{LM}, \mathrm{CZ}, \mathrm{CD}$, and $\mathrm{GY}$ prepared material, conducted experiments, collected data, and analyzed the data. ZL, LM and CC wrote the first draft of the manuscript. WW and CC guide the implementation of topics. All authors commented on previous versions of the manuscript. All authors read and approved the final manuscript.

\section{Funding}

The authors received no specific funding for this work.

\section{Availability of data and materials}

The data used to support the findings of this study are available from the corresponding author upon request.

\section{Declarations}

\section{Ethics approval and consent to participate}

The Ethics Committee of Renmin Hospital of Wuhan University approved the study. Patients all agreed to participate in the study.

\section{Consent for publication}

Not applicable.

\section{Competing interests}

There is no competing financial interest among authors.

\section{Author details}

'Department of General Surgery, Renmin Hospital of Wuhan University, No. 238, Jiefang Road, Wuchang District, Wuhan 430060, Hubei Province, China. ${ }^{2}$ Central Laboratory, Renmin Hospital of Wuhan University, No. 238, Jiefang Road, Wuchang District, Wuhan 430060, Hubei Province, China. ${ }^{3}$ Department of General Surgery, People's Hospital of Rizhao, 126 Tai'an Road, Donggang District, Rizhao 276800, Shandong Province, China.

Received: 10 November 2021 Accepted: 25 December 2021 Published online: 06 January 2022

\section{References}

1. Powers HJ. Riboflavin (vitamin B-2) and health. Am J Clin Nutr. 2003;77(6):1352-60

2. Powers HJ, Corfe BM, Nakano E. Riboflavin in development and cell fate. Subcell Biochem. 2012;56:229-45.

3. Bacher A, Eberhardt S, Fischer M, Kis K, Richter G. Biosynthesis of vitamin b2 (riboflavin). Annu Rev Nutr. 2000;20:153-67.
4. Institute of Medicine Standing Committee on the Scientific Evaluation of Dietary Reference I, its Panel on Folate OBV, Choline: The National Academies Collection: Reports funded by National Institutes of Health. In: Dietary Reference Intakes for Thiamin, Riboflavin, Niacin, Vitamin B(6), Folate, Vitamin B(12), Pantothenic Acid, Biotin, and Choline. edn. Washington (DC): National Academies Press (US). Copyright @ 1998, National Academy of Sciences.; 1998.

5. Yonezawa A, Inui K. Novel riboflavin transporter family RFVT/SLC52: identification, nomenclature, functional characterization and genetic diseases of RFVT/SLC52. Mol Aspects Med. 2013;34(2-3):693-701.

6. Console L, Tolomeo M, Colella M, Barile M, Indiveri C. Reconstitution in proteoliposomes of the recombinant human riboflavin transporter 2 (SLC52A2) overexpressed in E. coli. Int J Mol Sci. 2019;20(18):4416.

7. Yao Y, Yonezawa A, Yoshimatsu H, Masuda S, Katsura T, Inui K. Identification and comparative functional characterization of a new human riboflavin transporter hRFT3 expressed in the brain. J Nutr. 2010;140(7):1220-6.

8. Ericsson TA, Takeuchi Y, Templin C, Quinn G, Farhadian SF, Wood JC, Oldmixon BA, Suling KM, Ishii JK, Kitagawa Y, et al. Identification of receptors for pig endogenous retrovirus. Proc Natl Acad Sci USA. 2003;100(11):6759-64.

9. Foraker AB, Khantwal CM, Swaan PW. Current perspectives on the cellular uptake and trafficking of riboflavin. Adv Drug Deliv Rev. 2003;55(11):1467-83.

10. Cheng L, Zhang Q, Yang S, Yang Y, Zhang W, Gao H, Deng X, Zhang Q. A 4-gene panel as a marker at chromosome $8 \mathrm{q}$ in Asian gastric cancer patients. Genomics. 2013;102(4):323-30.

11. Ru B, Wong CN, Tong Y, Zhong JY, Zhong SSW, Wu WC, Chu KC, Wong CY, Lau CY, Chen I, et al. TISIDB: an integrated repository portal for tumorimmune system interactions. Bioinformatics. 2019;35(20):4200-2.

12. Gao J, Aksoy BA, Dogrusoz U, Dresdner G, Gross B, Sumer SO, Sun Y, Jacobsen A, Sinha R, Larsson E, et al. Integrative analysis of complex cancer genomics and clinical profiles using the cBioPortal. Sci Signal. 2013;6(269):1.

13. Tate JG, Bamford S, Jubb HC, Sondka Z, Beare DM, Bindal N, Boutselakis H, Cole CG, Creatore C, Dawson E, et al. COSMIC: the catalogue of somatic mutations in cancer. Nucleic Acids Res. 2019;47(D1):D941-d947.

14. Li T, Fan J, Wang B, Traugh N, Chen Q, Liu JS, Li B, Liu XS. TIMER: a web server for comprehensive analysis of tumor-infiltrating immune cells. Cancer Res. 2017;77(21):e108-10.

15. Li T, Fu J, Zeng Z, Cohen D, Li J, Chen Q, Li B, Liu XS. TIMER2.0 for analysis of tumor-infiltrating immune cells. Nucleic Acids Res. 2020;48(W1):W509-14.

16. Szklarczyk D, Franceschini A, Wyder S, Forslund K, Heller D, Huerta-Cepas J, Simonovic M, Roth A, Santos A, Tsafou KP, et al. STRING v10: protein-protein interaction networks, integrated over the tree of life. Nucleic Acids Res. 2015:43(Database issue):D447-52.

17. Li JH, Liu S, Zhou H, Qu LH, Yang JH. starBase v2.0: decoding miRNAceRNA, miRNA-ncRNA and protein-RNA interaction networks from largescale CLIP-Seq data. Nucleic Acids Res. 2014;42(Database issue):D92-97.

18. Thorsson V, Gibbs DL, Brown SD, Wolf D, Bortone DS, Ou Yang TH, PortaPardo E, Gao GF, Plaisier CL, Eddy JA, et al. The immune landscape of cancer. Immunity. 2019;51(2):411-2.

19. DeStefanis RA, Kratz JD, Emmerich PB, Deming DA. Targeted therapy in metastatic colorectal cancer: current standards and novel agents in review. Curr Colorectal Cancer Rep. 2019;15(2):61-9.

20. Aili A, Hasim A, Kelimu A, Guo X, Mamtimin B, Abudula A, Upur H. Association of the plasma and tissue riboflavin levels with C20orf54 expression in cervical lesions and its relationship to HPV16 infection. PLoS ONE. 2013;8(11):e79937.

21. Eli M, Li DS, Zhang WW, Kong B, Du CS, Wumar M, Mamtimin B, Sheyhidin I, Hasim A. Decreased blood riboflavin levels are correlated with defective expression of RFT2 gene in gastric cancer. World J Gastroenterol. 2012;18(24):3112-8

22. Vathipadiekal V, Wang V, Wei W, Waldron L, Drapkin R, Gillette M, Skates S, Birrer M. Creation of a human secretome: a novel composite library of human secreted proteins: validation using ovarian cancer gene expression data and a virtual secretome array. Clin Cancer Res. 2015;21(21):4960-9.

23. Tutino V, Defrancesco ML, Tolomeo M, Den V, Lorusso D, Paleni D, Caruso MG, Notarnicola M, Barile M. The expression of riboflavin transporters in human colorectal cancer. Anticancer Res. 2018;38(5):2659-67. 
24. Zhao Q, Wu C, Wang J, Li X, Fan Y, Gao S, Wang K. LncRNA SNHG3 promotes hepatocellular tumorigenesis by targeting miR-326. Tohoku J Exp Med. 2019;249(1):43-56.

25. Zhang PF, Wang F, Wu J, Wu Y, Huang W, Liu D, Huang XY, Zhang XM, Ke AW. LncRNA SNHG3 induces EMT and sorafenib resistance by modulating the miR-128/CD151 pathway in hepatocellular carcinoma. J Cell Physiol. 2019:234(3):2788-94.

26. Wu J, Zhang T, Chen Y, Ha S. MiR-139-5p influences hepatocellular carcinoma cell invasion and proliferation capacities via decreasing SLITRK4 expression. Biosci Rep. 2020;40(5).

27. Ito A, Kondo S, Tada K, Kitano S. Clinical development of immune checkpoint inhibitors. Biomed Res Int. 2015;2015:605478

28. Orabona C, Mondanelli G, Puccetti P, Grohmann U. Immune checkpoint molecules, personalized immunotherapy, and autoimmune diabetes. Trends Mol Med. 2018;24(11):931-41.

29. Darvin P, Toor SM, Sasidharan Nair V, Elkord E. Immune checkpoint inhibitors: recent progress and potential biomarkers. Exp Mol Med. 2018;50(12):1-11.

30. Rosenberg JE, Hoffman-Censits J, Powles T, van der Heijden MS, Balar AV, Necchi A, Dawson N, O'Donnell PH, Balmanoukian A, Loriot Y, et al. Atezolizumab in patients with locally advanced and metastatic urothelial carcinoma who have progressed following treatment with platinumbased chemotherapy: a single-arm, multicentre, phase 2 trial. Lancet. 2016;387(10031):1909-20.

31. Motzer RJ, Tannir NM, McDermott DF, Arén Frontera O, Melichar B, Choueiri TK, Plimack ER, Barthélémy P, Porta C, George S, et al. Nivolumab plus Ipilimumab versus sunitinib in advanced renal-cell carcinoma. N Engl J Med. 2018;378(14):1277-90.

32. Overman MJ, Lonardi S, Wong KYM, Lenz HJ, Gelsomino F, Aglietta M, Morse MA, Van Cutsem E, McDermott R, Hill A, et al. Durable clinical benefit with nivolumab plus ipilimumab in DNA mismatch repair-deficient/ microsatellite instability-high metastatic colorectal cancer. J Clin Oncol. 2018:36(8):773-9.

33. Sha D, Jin Z, Budczies J, Kluck K, Stenzinger A, Sinicrope FA. Tumor muta tional burden as a predictive biomarker in solid tumors. Cancer Discov. 2020:10(12):1808-25.

34. Yarchoan M, Hopkins A, Jaffee EM. Tumor mutational burden and response rate to PD-1 inhibition. N Engl J Med. 2017:377(25):2500-1.

35. Snyder A, Makarov V, Merghoub T, Yuan J, Zaretsky JM, Desrichard A, Walsh LA, Postow MA, Wong P, Ho TS, et al. Genetic basis for clinical response to CTLA-4 blockade in melanoma. N Engl J Med. 2014:371(23):2189-99.

36. Rizvi NA, Hellmann MD, Snyder A, Kvistborg P, Makarov V, Havel JJ, Lee W, Yuan J, Wong P, Ho TS, et al. Cancer immunology. Mutational landscape determines sensitivity to PD-1 blockade in non-small cell lung cancer. Science. 2015;348(6230):124-8.

37. Fumet JD, Truntzer C, Yarchoan M, Ghiringhelli F. Tumour mutational burden as a biomarker for immunotherapy: current data and emerging concepts. Eur J Cancer. 2020;131:40-50.

38. Samstein RM, Lee $C H$, Shoushtari AN, Hellmann MD, Shen R, Janjigian YY, Barron DA, Zehir A, Jordan EJ, Omuro A, et al. Tumor mutational load predicts survival after immunotherapy across multiple cancer types. Nat Genet. 2019;51(2):202-6.

39. Zhang CM, Lv JF, Gong L, Yu LY, Chen XP, Zhou HH, Fan L. Role of deficient mismatch repair in the personalized management of colorectal cancer. Int J Environ Res Public Health. 2016;13(9):892.

40. Shen H, Zhong M, Wang W, Liao P, Yin X, Rotroff D, Knepper TC, McLeod HL, Zhou C, Xie S, et al. EBV infection and MSI status significantly influence the clinical outcomes of gastric cancer patients. Clin Chim Acta. 2017:471:216-21

41. Zhao P, Li L, Jiang X, Li Q. Mismatch repair deficiency/microsatellite instability-high as a predictor for anti-PD-1/PD-L1 immunotherapy efficacy. J Hematol Oncol. 2019;12(1):54.

42. Zhang $Y$, Zhang Z. The history and advances in cancer immunotherapy: understanding the characteristics of tumor-infiltrating immune cells and their therapeutic implications. Cell Mol Immunol. 2020;17(8):807-21.

43. Cassetta L, Pollard JW. Targeting macrophages: therapeutic approaches in cancer. Nat Rev Drug Discov. 2018;17(12):887-904.

44. Ruffell B, Coussens LM. Macrophages and therapeutic resistance in cancer. Cancer Cell. 2015;27(4):462-72.
45. Relation T, Yi T, Guess AJ, La Perle K, Otsuru S, Hasgur S, Dominici M, Breuer C, Horwitz EM. Intratumoral delivery of interferony-secreting mesenchymal stromal cells repolarizes tumor-associated macrophages and suppresses neuroblastoma proliferation in vivo. Stem Cells. 2018;36(6):915-24.

46. Han C, Yang Y, Sheng Y, Wang J, Li W, Zhou X, Guo L. The mechanism of IncRNA-CRNDE in regulating tumour-associated macrophage M2 polarization and promoting tumor angiogenesis. J Cell Mol Med. 2021:25(9):4235-47.

47. Mantovani A, Sozzani S, Locati M, Allavena P, Sica A. Macrophage polarization: tumor-associated macrophages as a paradigm for polarized M2 mononuclear phagocytes. Trends Immunol. 2002;23(11):549-55.

48. Pyonteck SM, Akkari L, Schuhmacher AJ, Bowman RL, Sevenich L, Quail DF, Olson OC, Quick ML, Huse JT, Teijeiro V, et al. CSF-1R inhibition alters macrophage polarization and blocks glioma progression. Nat Med. 2013;19(10):1264-72.

\section{Publisher's Note}

Springer Nature remains neutral with regard to jurisdictional claims in published maps and institutional affiliations.
Ready to submit your research? Choose BMC and benefit from:

- fast, convenient online submission

- thorough peer review by experienced researchers in your field

- rapid publication on acceptance

- support for research data, including large and complex data types

- gold Open Access which fosters wider collaboration and increased citations

- maximum visibility for your research: over 100M website views per year

At BMC, research is always in progress.

Learn more biomedcentral.com/submissions 